\title{
Optical properties of InAsBi and optimal designs of lattice-matched and strain- balanced III-V semiconductor superlattices
}

P. T. Webster, A. J. Shalindar, N. A. Riordan, C. Gogineni, H. Liang, A. R. Sharma, and S. R. Johnson

Citation: Journal of Applied Physics 119, 225701 (2016); doi: 10.1063/1.4953027

View online: http://dx.doi.org/10.1063/1.4953027

View Table of Contents: http://aip.scitation.org/toc/jap/119/22

Published by the American Institute of Physics

\section{Articles you may be interested in}

Measurement of InAsBi mole fraction and InBi lattice constant using Rutherford backscattering spectrometry and X-ray diffraction

Journal of Applied Physics 120, 145704 (2016); 10.1063/1.4964799

Measurement of InAsSb bandgap energy and InAs/InAsSb band edge positions using spectroscopic ellipsometry and photoluminescence spectroscopy

Journal of Applied Physics 118, 245706 (2015); 10.1063/1.4939293

Bi-induced band gap reduction in epitaxial InSbBi alloys

Applied Physics Letters 105, 212101 (2014); 10.1063/1.4902442

Demonstration of InAsBi photoresponse beyond $3.5 \mu \mathrm{m}$

Applied Physics Letters 104, 171109 (2014); 10.1063/1.4873403

Absorption properties of type-II InAs/InAsSb superlattices measured by spectroscopic ellipsometry

Applied Physics Letters 106, 061907 (2015); 10.1063/1.4908255

8-band and 14-band kp modeling of electronic band structure and material gain in $\mathrm{Ga}(\mathrm{In}) \mathrm{AsBi}$ quantum wells grown on GaAs and InP substrates

Journal of Applied Physics 118, 055702 (2015); 10.1063/1.4927922

\section{AlP | Journal of}

Save your money for your research.

It's now FREE to publish with us no page, color or publication charges apply.
Publish your research in the

Joumal of Applied Physics

to claim your place in applied

physics history. 


\title{
Optical properties of InAsBi and optimal designs of lattice-matched and strain-balanced III-V semiconductor superlattices
}

\author{
P. T. Webster,${ }^{1, a)}$ A. J. Shalindar, ${ }^{2}$ N. A. Riordan, ${ }^{1}$ C. Gogineni, ${ }^{1}$ H. Liang, ${ }^{1}$ A. R. Sharma,${ }^{1}$ \\ and S. R. Johnson ${ }^{1}$ \\ ${ }^{1}$ Center for Photonics Innovation and School of Electrical, Computer, and Energy Engineering, Arizona State \\ University, Tempe, Arizona 85287, USA \\ ${ }^{2}$ Center for Photonics Innovation and School for Engineering of Matter, Transport, and Energy, Arizona State \\ University, Tempe, Arizona 85287, USA
}

(Received 19 April 2016; accepted 16 May 2016; published online 8 June 2016)

\begin{abstract}
The optical properties of bulk $\operatorname{InAs} \mathrm{As}_{0.936} \mathrm{Bi}_{0.064}$ grown by molecular beam epitaxy on a (100)oriented $\mathrm{GaSb}$ substrate are measured using spectroscopic ellipsometry. The index of refraction and absorption coefficient are measured over photon energies ranging from $44 \mathrm{meV}$ to $4.4 \mathrm{eV}$ and are used to identify the room temperature bandgap energy of bulk $\operatorname{InAs} \mathrm{s}_{0.936} \mathrm{Bi}_{0.064}$ as $60.6 \mathrm{meV}$. The bandgap of InAsBi is expressed as a function of $\mathrm{Bi}$ mole fraction using the band anticrossing model and a characteristic coupling strength of $1.529 \mathrm{eV}$ between the Bi impurity state and the InAs valence band. These results are programmed into a software tool that calculates the miniband structure of semiconductor superlattices and identifies optimal designs in terms of maximizing the electron-hole wavefunction overlap as a function of transition energy. These functionalities are demonstrated by mapping the design spaces of lattice-matched $\mathrm{GaSb} / \mathrm{InAs}_{0.911} \mathrm{Sb}_{0.089}$ and $\mathrm{GaSb} /$ $\mathrm{InAs}_{0.932} \mathrm{Bi}_{0.068}$ and strain-balanced InAs/InAsSb, InAs/GaInSb, and InAs/InAsBi superlattices on $\mathrm{GaSb}$. The absorption properties of each of these material systems are directly compared by relating the wavefunction overlap square to the absorption coefficient of each optimized design. Optimal design criteria are provided for key detector wavelengths for each superlattice system. The optimal design mid-wave infrared InAs/InAsSb superlattice is grown using molecular beam epitaxy, and its optical properties are evaluated using spectroscopic ellipsometry and photoluminescence spectroscopy. Published by AIP Publishing. [http://dx.doi.org/10.1063/1.4953027]
\end{abstract}

\section{INTRODUCTION}

For many decades since the first proposal to utilize quantum wells to modify the optoelectronic properties of semiconductor materials, ${ }^{1}$ band structure engineering has been a technologically valuable field of research. The need for engineered materials can be readily seen in the plots of the bandgap energy as a function of the lattice constant of selected III-V semiconductors ${ }^{2}$ shown in Figs. 1(a) at $0 \mathrm{~K}$ and 1(b) at $295 \mathrm{~K}$. Direct (indirect) bandgaps are indicated by solid circles (squares) and solid (dotted) curves for binary and ternary alloys, respectively. Though the bandgap energies of the ternary alloys presented span a continuous spectral range, only limited compositional ranges can be grown in thick layers with high epitaxial quality due to the lack of commonly available lattice-matched substrates (indicated with vertical dashed lines) at every lattice constant. The main challenge to optoelectronic device designers has always been in finding materials with appropriate bandgap energies for the desired application, which also have lattice constants near those of commercially available substrates.

Alloying bismuth into InAs provides one possible means of reaching longer wavelengths. Photoluminescence measurements of bulk InAsBi samples grown by metalorganic

${ }^{\text {a)} E l e c t r o n i c ~ m a i l: ~ p r e s t o n . t . w e b s t e r @ a s u . e d u ~}$ vapor phase epitaxy indicate that the bandgap energy decreases at a rate of 42 to $55 \mathrm{meV} / \% \mathrm{Bi}^{3,4}$ and droplet-free InAsBi has been grown nearly lattice-matched on GaSb substrate by molecular beam epitaxy. ${ }^{5}$ Nevertheless, ternary alloys provide relatively little bandgap engineering flexibility due to the constraint that high quality material needs to be nearly lattice-matched, drastically limiting the range of mole fractions that can be grown dislocation free on a given substrate. An additional degree of design freedom is necessary to simultaneously select the bandgap energy and lattice constant; this can be achieved using a quaternary alloy or by engineering the band structure using a superlattice. ${ }^{6}$

For a superlattice composed of a binary and a ternary alloy, the ternary composition and the individual layer thicknesses of the two repeating layers constitute three design parameters. However, in order to grow an arbitrarily thick superlattice region without strain relaxation, either a latticematched or strain-balanced superlattice must be utilized; ${ }^{7}$ this reduces the number of free design parameters to two. In the case of lattice-matched structures, the two free parameters are the thickness of each of the two constituent layers, and in the case of strain-balanced structures, the two free parameters are the ternary layer composition and the superlattice period thickness. One of the two remaining design parameters is required to tune the ground state transition energy, leaving the second parameter available for design 

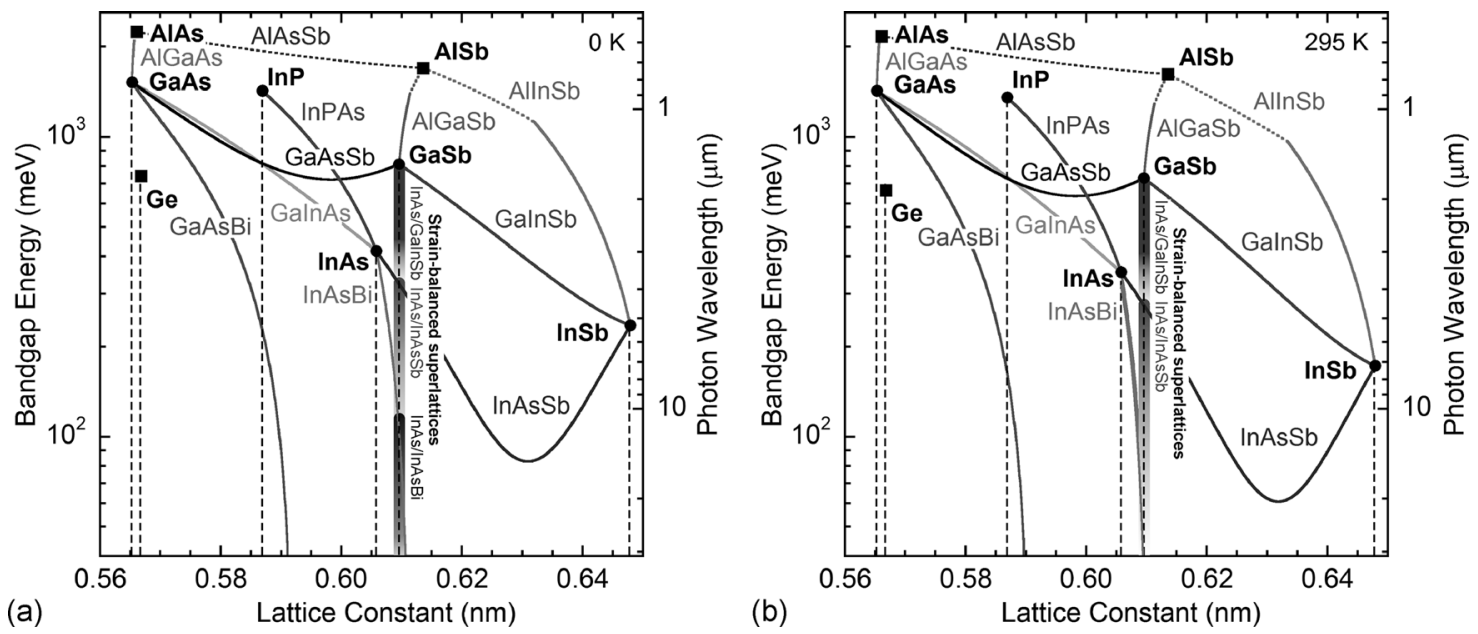

FIG. 1. Bandgap energy as a function of the lattice constant for selected III-V binary and ternary alloys with the lattice constants of common substrates indicated with vertical dashed lines; (a) low temperature and (b) room temperature. Lattice-matched InAsSb and InAsBi and strain-balanced InAs/GaInSb, InAs/ InAsSb, and InAs/InAsBi superlattices can be grown on GaSb. For each superlattice system, the vertical lines with dark to light color-gradient show the spectral range and the reduction in electron-hole wavefunction overlap as longer wavelength cutoffs are attained.

optimization. The absorption coefficient of the superlattice is proportional to the square of the electron-hole wavefunction overlap, ${ }^{8}$ thus in a superlattice system with at least two free design parameters the absorption strength can be optimized as a function of detector wavelength.

For a strain-balanced superlattice composed of two ternary alloys, the second alloy composition can act as a third design parameter. A third degree of design freedom could be used to optimize another characteristic of the superlattice, such as the density of states. No additional design freedom is gained by using ternary alloys in the lattice-matched superlattice; however lattice-matched quaternaries would provide additional design freedom.

In this work, a software tool is developed that calculates the design spaces of lattice-matched and strain-balanced semiconductor superlattices, and identifies the optimal designs in terms of maximizing wavefunction overlap as a function of ground state transition energy. This technique is used to calculate the design spaces of lattice-matched $\mathrm{GaSb} / \mathrm{InAs}_{0.911} \mathrm{Sb}_{0.089}$ and $\mathrm{GaSb} / \mathrm{InAs}_{0.932} \mathrm{Bi}_{0.068}$ superlattices and strain-balanced InAs/InAsSb, InAs/GaInSb, and InAs/InAsBi superlattices on $\mathrm{GaSb}$ substrates at low temperature and at room temperature. In all five superlattice systems considered, the ground state transition occurs between the first electron and first heavy hole minibands. These material systems are compared by relating the wavefunction overlap square to the absorption coefficient of each optimized design, and temperature dependences are discussed.

\section{SPECTROSCOPIC ELLIPSOMETRY OF $\operatorname{InAs} s_{0.936} \mathrm{Bi}_{0.064}$}

The bandgap and absorption properties of InAsBi are determined from a $930 \mathrm{~nm}$ thick layer of bulk $\operatorname{InAs} \mathrm{As}_{0.936} \mathrm{Bi}_{0.064}$ grown by molecular beam epitaxy on a (100)-oriented $50 \mathrm{~mm}$ GaSb substrate. ${ }^{5,9}$ The sample structure consists of a $500 \mathrm{~nm}$ thick GaSb buffer, a $13.95 \mathrm{~nm}$ thick InAs layer, a $930 \mathrm{~nm}$ thick $\operatorname{InAs}_{0.936} \mathrm{Bi}_{0.064}$ layer, and a $9.30 \mathrm{~nm}$ thick InAs cap, and is shown in Fig. 2. A smooth, droplet-free surface is maintained throughout the growth using a constant 0.5 monolayer per second In-limited growth rate, an As/In flux ratio of 0.98 to 0.99 , a $\mathrm{Bi} / \mathrm{In}$ flux ratio of 0.065 , and a growth temperature of $280^{\circ} \mathrm{C}$ calibrated using an Ircon Modline 3 (model 3G10C05) infrared pyrometer. ${ }^{10}$ Reciprocal space mapping around the (115)-plane diffraction verifies that the sample is coherently strained on the GaSb substrate. X-ray diffraction from the (004)-planes provides the Bi mole fraction of 0.0639 and the inferred tensile strain of $0.036 \%{ }^{9}$

The optical properties of the $\operatorname{InAs}_{0.936} \mathrm{Bi}_{0.064}$ layer are measured by spectroscopic ellipsometry using VASE and IR-VASE model spectroscopic ellipsometers to, respectively, cover the ultraviolet to near infrared and the near infrared to long wavelength infrared spectral ranges. ${ }^{11}$ The ellipsometric parameters $\Psi$ and $\Delta$ are measured at $64^{\circ}, 68^{\circ}$, $72^{\circ}$, and $76^{\circ}$ and are identical over the spectral range that the two ellipsometers overlap ( 0.6 to $0.8 \mathrm{eV})$. Combining the

\begin{tabular}{|c|c|}
\hline & $1.00 \mathrm{~nm} \ln A \mathrm{~s}$ oxide \\
\hline $9.30 \mathrm{~nm} \ln A s$ & $9.30 \mathrm{~nm} \ln A \mathrm{~s}$ \\
\hline $\begin{array}{c}930 \mathrm{~nm} \\
\ln \mathrm{As}_{0.936} \mathrm{Bi}_{0.064}\end{array}$ & $\begin{array}{c}930 \mathrm{~nm} \\
\text { In } \mathrm{As}_{0.936} \mathrm{Bi}_{0.064} \\
\text { (optical constants fit) }\end{array}$ \\
\hline $13.95 \mathrm{~nm} \ln A s$ & $13.95 \mathrm{~nm} \ln A \mathrm{~s}$ \\
\hline $500 \mathrm{~nm} \mathrm{GaSb}$ & \multirow{2}{*}{$\begin{array}{c}\text { GaSb } \\
\text { Substrate }\end{array}$} \\
\hline $\begin{array}{l}\text { p-type GaSb } \\
\text { Substrate }\end{array}$ & \\
\hline
\end{tabular}

FIG. 2. Bulk InAsBi sample cross section (left) alongside the optical model used for ellipsometry analysis (right) in which the optical constants of the $930 \mathrm{~nm}$ thick $\mathrm{InAs}_{0.936} \mathrm{Bi}_{0.064}$ layer are fit to the measured ellipsometric parameters. 
data measured by the two ellipsometers, the ellipsometric parameters are obtained for photon energies ranging from $44 \mathrm{meV}$ to $4.4 \mathrm{eV}$ ( 28.2 to $0.282 \mu \mathrm{m}$ wavelength). The depolarization of the measured reflection is reduced below $2 \%$ by roughing the backside of the semi-transparent $\mathrm{GaSb}$ wafer with 320 grit sandpaper prior to measurement.

The ellipsometric model of the sample is shown schematically alongside the physical cross section in Fig. 2. The WVASE software library provides the optical constants of the GaSb, InAs, and InAs native oxide layers, ${ }^{12}$ and the optical constants of the InAsBi layer are determined using the point-by-point analysis method that generates a pair of raw optical constants for each wavelength measured. ${ }^{13}$ The results are shown as the solid black curves in Fig. 3, where the refractive index $n$ (left-hand vertical axis) and extinction coefficient $k$ (right-hand vertical axis) are plotted as a function of photon energy $h \nu$ (lower horizontal axis) and photon wavelength $\lambda$ (upper horizontal axis). A general oscillator model composed of a PSEMIO oscillator at the bandgap and Gaussian oscillators above the bandgap ${ }^{12}$ is fit to the raw data and shown as grey curves in Fig. 3; the oscillator model fit to the $n$ and $k$ data confirms that the raw (point-by-point) optical constants are Kramers-Kronig consistent.

The absorption coefficient $\alpha=4 \pi k / \lambda$ shown in Fig. 4 is determined using the raw extinction coefficient $(k)$ data in Fig. 3. The figure inset shows the absorption coefficient in the range of 50 to $100 \mathrm{meV}$ (black curve) and its first derivative (grey curve). The position of the first derivative maximum identifies the tensilely strained $\operatorname{InAs}_{0.936} \mathrm{Bi}_{0.064}$ bandgap energy of $60 \mathrm{meV}$ with an absorption coefficient of $503 \mathrm{~cm}^{-1}$.

\section{BAND ANTICROSSING IN InAsBi}

The valence band edge of $\operatorname{InAs}_{(1-x)} \mathrm{Bi}_{x}$ is expressed as a function of Bi mole fraction $x$ using the valence band anticrossing model in Equation (1). ${ }^{14,15}$ In the equation, $E_{v}(x)$ is the position of the InAsBi valence band edge relative to the position of the InAs valence band edge; $\Delta E_{v}$ is the energy separation between the InBi and InAs valence band edges; $E_{B i}$ is the energy of the Bi impurity state relative to the InAs valence band edge; and $C_{B i}$ is the coupling parameter that characterizes the strength of the interaction between the Bi impurity state and the InAs valence band. Physically, the quantity inside the first set of square brackets represents the valence band anticrossing interaction which dominates in the As-rich mole fraction regime, while the quantity in the second set of square brackets represents the linear variation in the valence band which is assumed to dominate in the Bi-rich mole fraction regime as is the case for $\mathrm{Sb}$ in $\mathrm{GaAsSb}^{14}$ and $\mathrm{InAsSb}^{16}$ and is similarly assumed for $\mathrm{Bi}$ in GaAsBi. ${ }^{14}$ Correspondingly, the As constituent could produce an anticrossing interaction in the conduction band for Bi-rich mole fractions; ${ }^{14,16}$ however, since Bi-rich InAsBi has not been synthesized and measured, the conduction band edge is assumed to vary linearly with mole fraction between the conduction band edges of InAs and InBi.

$$
E_{v}(x)=\left[\frac{\left(\Delta E_{v} \cdot x\right)+E_{B i}+\sqrt{\left(\left(\Delta E_{v} \cdot x\right)-E_{B i}\right)^{2}+\left(2 C_{B i}\right)^{2} \cdot x}}{2}\right](1-x)+\left[\Delta E_{v} \cdot x\right] x .
$$

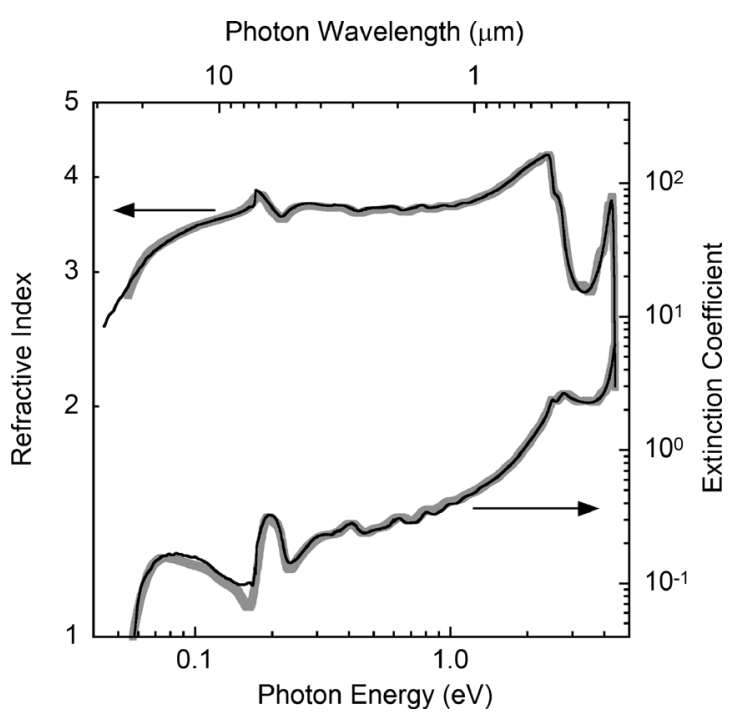

FIG. 3. Refractive index (left-hand vertical axis) and extinction coefficient (right-hand vertical axis) of bulk InAs ${ }_{0.936} \mathrm{Bi}_{0.064}$ plotted as a function of photon energy (lower horizontal axis) and photon wavelength (upper horizontal axis). The optical constants identified using point-by-point and general oscillator fitting analyses are shown with black and grey curves, respectively.
The valence band edge of InBi is predicted by linearly extrapolating from the valence band edges of InP, InAs, and $\mathrm{InSb}$ as a function of lattice constant ${ }^{2}$ in Fig. 5, from which the energy separation between the InBi and InAs valence band edges, $\Delta E_{v}$, of $0.80 \mathrm{eV}$ is identified at the $0.66108 \mathrm{~nm}$ lattice constant of InBi. ${ }^{9}$ The conduction band edge of InBi is specified by the InBi valence band edge plus the $-1.63 \mathrm{eV}$ bandgap energy of InBi calculated using density functional theory. ${ }^{17}$ The Bi impurity state is located $0.4 \mathrm{eV}$ below the valence band edge of $\mathrm{GaAs}^{14}$ which is itself located $0.21 \mathrm{eV}$ below the valence band edge of InAs; ${ }^{2}$ therefore, the energy of the $\mathrm{Bi}$ impurity state relative to the InAs valence band edge, $E_{B i}$, is $-0.61 \mathrm{eV}$.

When InAsBi is grown pseudomorphically on $\mathrm{GaSb}$ as is the case for the bulk $\operatorname{InAs}_{0.936} \mathrm{Bi}_{0.064}$ layer examined in Section II, strain modifies the conduction and valence band edge positions of the material. The band anticrossing model characterizes the band edge positions of unstrained material; therefore, the coupling parameter $C_{B i}$ in Equation (1) is fit using the unstrained $\operatorname{InAs}_{0.936} \mathrm{Bi}_{0.064}$ bandgap specified by the $60 \mathrm{meV}$ strained bandgap measured in Section II plus $0.6 \mathrm{meV}$ due to the $0.036 \%$ tensile strain in the layer. The 


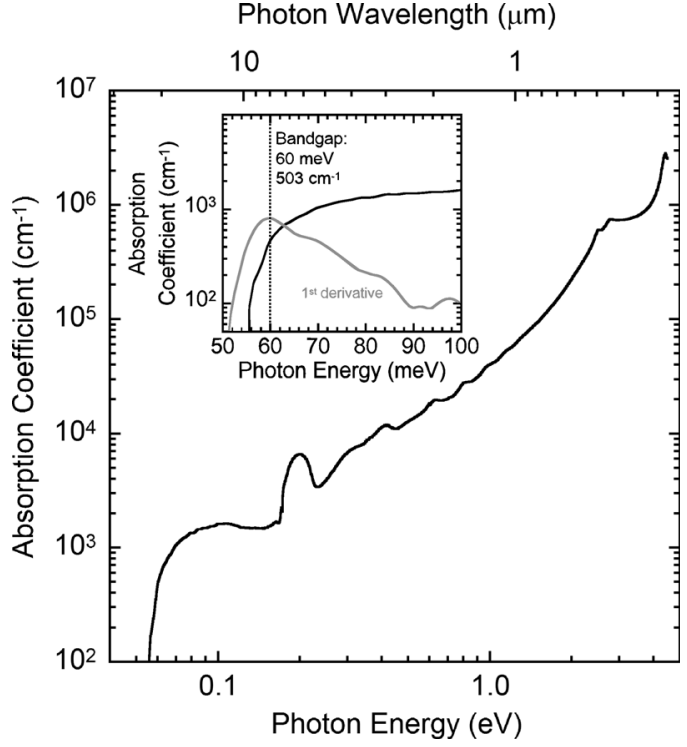

FIG. 4. Absorption coefficient of $\operatorname{InAs}_{0.936} \mathrm{Bi}_{0.064}$ plotted as a function of photon energy (lower horizontal axis) and photon wavelength (upper horizontal axis). Inset shows the absorption coefficient in the range of 50 to $100 \mathrm{meV}$ (black curve) as well as its first derivative (grey curve) that is used to determine the bandgap energy.

$0.6 \mathrm{meV}$ strain offset is calculated using the Pikus-Bir Hamiltonian, ${ }^{18,19}$ the deformation potentials of InAs, and a composition-dependent Poisson's ratio which varies linearly with mole fraction between Poisson's ratio of InAs (0.352) and InBi (0.350). ${ }^{2,9}$

The $\operatorname{InAs}_{(1-x)} \mathrm{Bi}_{x}$ bandgap energy as a function of $\mathrm{Bi}$ mole fraction is computed using the valence band anticrossing model and the Bi coupling parameter and other parameters given in Tables I and II, and is shown in Fig. 6. The unstrained bulk bandgap is shown by the solid grey curve. When this material is coherently strained to GaSb (strain displayed on the upper horizontal axis), the light and heavy hole bands split resulting in light hole (dotted black curve) and heavy hole (solid black curve) bandgaps. InAsBi is

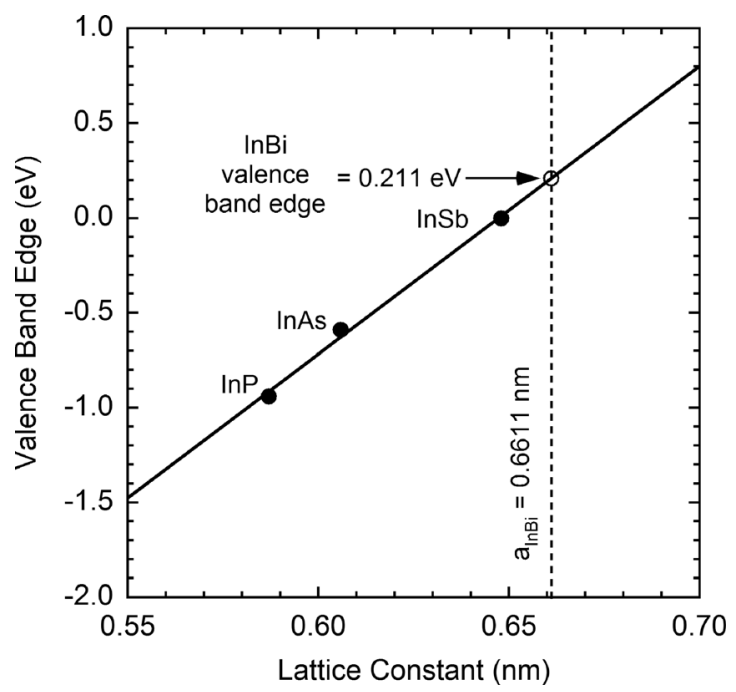

FIG. 5. Valence band edges of InP, InAs, and InSb (solid circles) plotted as a function of lattice constant. The solid line is a linear fit to the data that is used to predict the valence band edge of InBi (open circle).
TABLE I. InAsBi valence band anticrossing model parameters.

\begin{tabular}{lc}
\hline \hline Parameter & Value $(\mathrm{eV})$ \\
\hline Energy separation between the InAs and & 0.80 \\
InBi valence band edges, $\Delta E_{v}$ & \\
Bi impurity level, $E_{B i}$ & -0.61 \\
Bi coupling parameter, $C_{B i}$ & 1.529 \\
\hline \hline
\end{tabular}

perfectly lattice-matched to $\mathrm{GaSb}$ at $6.8 \% \mathrm{Bi}$ and becomes semimetallic at $8.0 \% \mathrm{Bi}$ at room temperature. The conduction and valence band edges $\left(E_{c}\right.$ and $\left.E_{v}\right)$ of tensilely strained InAs and lattice-matched $\operatorname{InAs}_{0.932} \mathrm{Bi}_{0.068}$ on $\mathrm{GaSb}$ are shown in the inset. Over the $0 \%$ to $3 \% \mathrm{Bi}$ mole fraction range of bulk InAsBi, the average linear bulk bandgap reduction rate per unit mole fraction is $50.9 \mathrm{meV} / \% \mathrm{Bi}$, which lies between the rates of 42 and $55 \mathrm{meV} / \% \mathrm{Bi}$ reported over the

TABLE II. Physical and band structure parameters of InAs and InBi.

\begin{tabular}{lcc}
\hline \hline & \multicolumn{2}{c}{ Value } \\
\cline { 2 - 3 } Parameter & InAs & InBi \\
\hline Valence band edge & $-0.59 \mathrm{eV}$ & $0.21 \mathrm{eV}$ \\
0 K bandgap energy & $0.417 \mathrm{eV}$ & $-1.63 \mathrm{eV}$ \\
295 K bandgap energy & $0.354 \mathrm{eV}$ & $-1.63 \mathrm{eV}$ \\
Lattice constant & $0.60583 \mathrm{~nm}$ & $0.66108 \mathrm{~nm}$ \\
Poisson's ratio & 0.352 & 0.350 \\
Conduction band deformation potential & $-5.08 \mathrm{eV}$ & $-5.08 \mathrm{eV}$ \\
Valence band deformation potential & $-1.00 \mathrm{eV}$ & $-1.00 \mathrm{eV}$ \\
Shear deformation potential & $-1.80 \mathrm{eV}$ & $-1.80 \mathrm{eV}$ \\
Electron effective mass & 0.026 & 0.026 \\
Heavy hole effective mass & 0.333 & 0.333 \\
\hline \hline
\end{tabular}

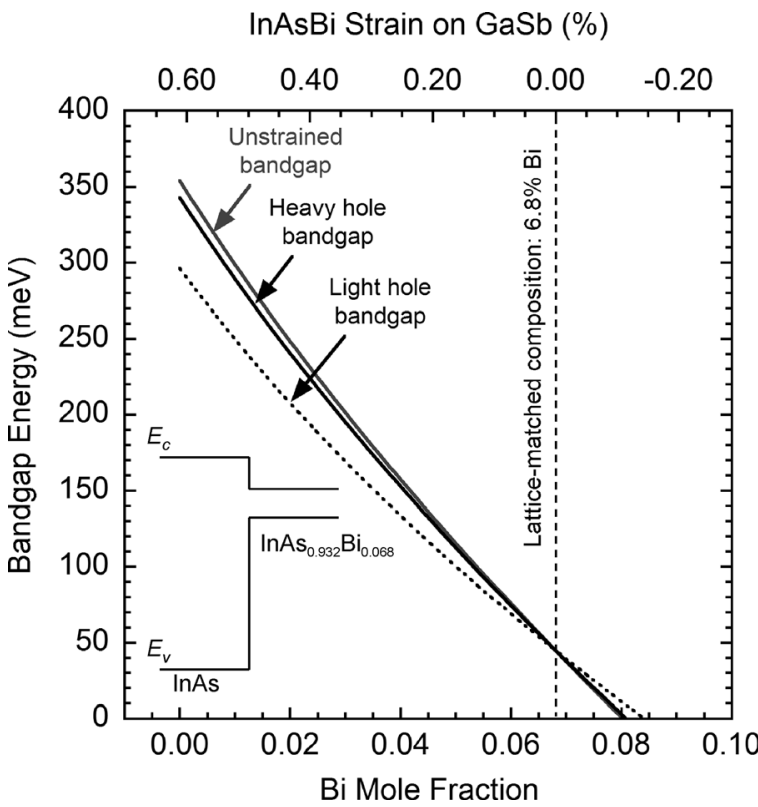

FIG. 6. Room temperature bandgap energy of InAsBi as a function of $\mathrm{Bi}$ mole fraction (lower horizontal axis). The solid grey curve shows the unstrained bandgap and the dotted and solid black curves show the light and heavy hole bandgaps of coherently strained InAsBi on GaSb. The corresponding strain is shown on the upper horizontal axis. The inset shows the conduction and valence band edge positions $\left(E_{c}\right.$ and $\left.E_{v}\right)$ of tensilely strained InAs and lattice-matched $\operatorname{InAs}_{0.932} \mathrm{Bi}_{0.068}$ on $\mathrm{GaSb}$. 
same mole fraction range in Refs. 3 and 4. For the strained material, the bandgap reduction rate is $42.4 \mathrm{meV} / \% \mathrm{Bi}$ over the same range of mole fractions ( $0 \%$ to $3 \% \mathrm{Bi})$.

\section{OPTIMAL DESIGN SOFTWARE}

The bulk material parameters needed to calculate the superlattice miniband structure are the bandgap energy, valence band edge, lattice constant, Poisson's ratio, deformation potentials, and effective masses of each of the superlattice constituents. The design calculations utilize the III-V semiconductor material parameters for InAs, GaSb, InAsSb, and GaInSb taken from a review article ${ }^{2}$ published in 2001, along with other parameters detailed below.

The bandgaps and valence band edges of InAsSb and GaInSb are characterized by bowing model relationships. The bandgap bowing parameter of InAsSb is $938 \mathrm{meV}$ at low temperature and $750 \mathrm{meV}$ at room temperature, and the valence band bowing parameter of InAsSb is $-380 \mathrm{meV}$ at low temperature and $-367 \mathrm{meV}$ at room temperature. ${ }^{20}$ The bandgap bowing parameter of $\mathrm{GaInSb}$ is $413 \mathrm{meV}$ at low temperature and $398 \mathrm{meV}$ at room temperature. ${ }^{21}$ The valence band bowing parameter of GaInSb is zero implying that the bandgap bowing occurs entirely in the conduction band of GaInSb; no temperature dependence has been reported. ${ }^{2}$ The InAsBi material parameters are discussed in Section III and outlined in Tables I and II; the Bi coupling parameter is assumed to be independent of temperature to calculate the InAs/InAsBi band alignment at low temperature.

The strained band alignment of each superlattice is established using the Pikus-Bir Hamiltonian, ${ }^{18,19}$ and the electron, heavy hole, and light hole miniband energies and wavefunctions are calculated using the Kronig-Penney model. ${ }^{22-24}$ The ground state transition energy (effective bandgap) and wavefunction overlap square (quantity proportional to absorption coefficient $)^{8}$ are determined as a function of the free design parameters, and these key aspects of each superlattice system are presented graphically in figures referred to as the design space maps. For strain-balanced material systems, the ground state transition energy is plotted on the vertical axis, alloy composition is plotted on the horizontal axis, and contours of constant period thickness are displayed in the plot. Similar figures are generated for lattice-matched systems, except that the horizontal axis is represented by one of the two constituent layer thicknesses, generally the larger bandgap material for figure clarity. By overlaying contours of constant wavefunction overlap square onto the figure, optimal superlattice designs are readily identified as a function of ground state transition energy.

The optimal superlattice designs in this work have been calculated at low temperature and at room temperature, and the optimal design criteria are provided for the technologically important 3 to 5 and 8 to $12 \mu \mathrm{m}$ wavelength atmospheric transmission windows. All material parameters utilized in these calculations are provided in Appendix A.

\section{LATTICE-MATCHED GaSb/InAsSb AND GaSb/ InAsBi SUPERLATTICES}

The low and room temperature design space maps of the lattice-matched $\mathrm{GaSb} / \mathrm{InAs}_{0.911} \mathrm{Sb}_{0.089}$ superlattice system are presented in Figs. 7(a) at $0 \mathrm{~K}$ and 7 (b) at $295 \mathrm{~K}$, which plot the ground state transition energy of the superlattice (solid curves) as a function of the GaSb layer thickness for period thicknesses of $1,3,5,8,10,12$, and $15 \mathrm{~nm}$. The bandgap energies of $\mathrm{GaSb}$ and $\mathrm{InAs}_{0.911} \mathrm{Sb}_{0.089}$ are indicated in the figures as horizontal dash-dot lines. As the GaSb layer thickness approaches zero, the ground state transition energy of the superlattice approaches the bandgap energy of In $\mathrm{As}_{0.911} \mathrm{Sb}_{0.089}$, and when the $\mathrm{InAs}_{0.911} \mathrm{Sb}_{0.089}$ layer thickness approaches zero, the ground state transition energy of the superlattice approaches the bandgap energy of GaSb. For intermediate layer thicknesses, transition energies are obtained anywhere between the bandgap energy of GaSb and zero, and even negative (semimetallic) transitions; a result of the type-II broken gap band alignment between $\mathrm{GaSb}$ and $\operatorname{InAs}_{0.911} \mathrm{Sb}_{0.089}$.

In order to identify which design will provide maximum absorption for a given transition energy, contours of constant wavefunction overlap squared (dotted curves) are overlaid
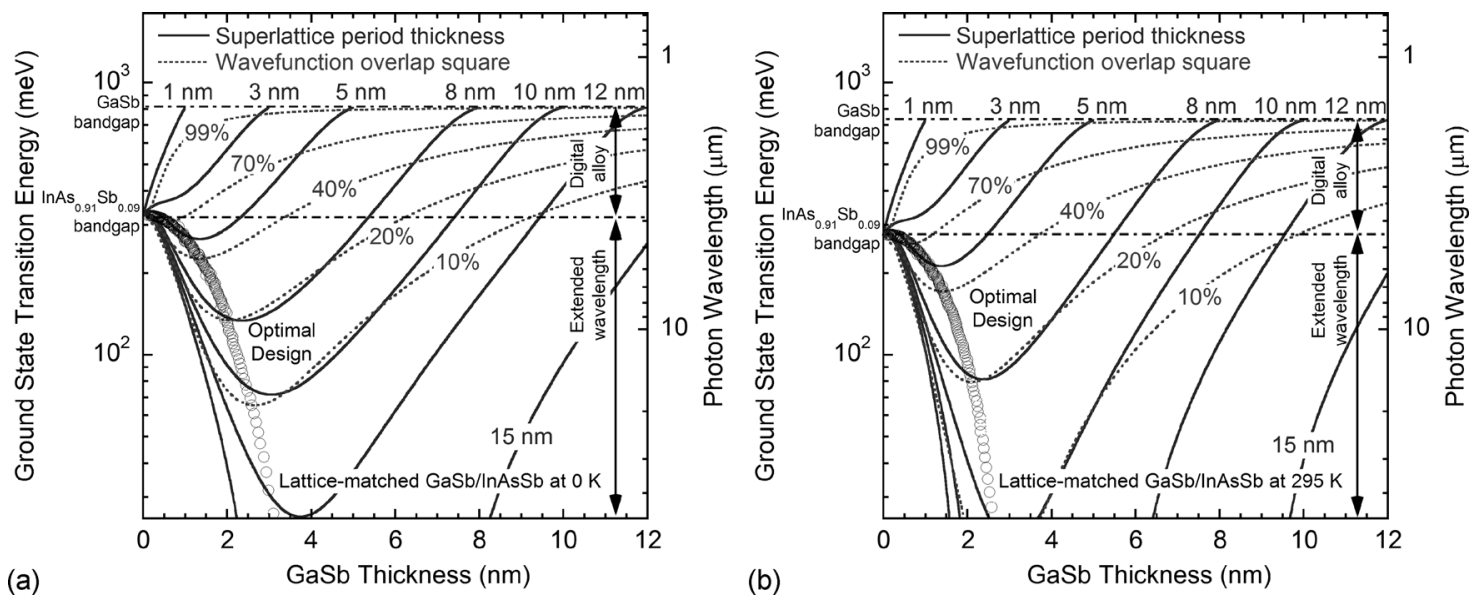

FIG. 7. Ground state transition energy of lattice-matched $\mathrm{GaSb} / \mathrm{InAs}_{0.911} \mathrm{Sb}_{0.089}$ superlattices as a function of GaSb layer thickness; (a) low temperature and (b) room temperature. The solid curves are contours of constant superlattice period thickness. The dotted curves are contours of constant electron-hole wavefunction overlap squared expressed as a percentage. The minima in the wavefunction overlap square contours represent designs that provide maximum absorption for a given transition energy shown by the open circles. 
onto the figures. $100 \%$ wavefunction overlap occurs at the bandgap energies of $\mathrm{GaSb}$ and $\mathrm{InAs}_{0.911} \mathrm{Sb}_{0.089}$ representing limiting cases that arise when one layer constituent comprises the full period thickness of the superlattice. Between the bandgap energies of $\mathrm{GaSb}$ and $\mathrm{InAs}_{0.911} \mathrm{Sb}_{0.089}$, the wavefunction overlap increases with decreasing period thickness and $100 \%$ wavefunction overlap occurs in the limit that the period thickness approaches zero. This region of the design space is defined as the digital alloy regime because the optimal design for a cutoff in this spectral range is an infinitesimally short period superlattice that is a digital ana$\log ^{25,26}$ of the lattice-matched random quaternary alloy $(\mathrm{GaSb})_{1-\mathrm{z}}\left(\mathrm{InAs}_{0.911} \mathrm{Sb}_{0.089}\right)_{\mathrm{z}}$ that covers the same range with $100 \%$ wavefunction overlap. ${ }^{2}$

Perhaps more interesting, however, are the superlattice designs below the bandgap energy of $\operatorname{InAs}_{0.911} \mathrm{Sb}_{0.089}$, much of which cannot be accessed using lattice-matched $(\mathrm{GaSb})_{1-\mathrm{z}}\left(\mathrm{InAs}_{0.911} \mathrm{Sb}_{0.089}\right)_{\mathrm{z}}$. In this region, defined as the extended wavelength regime, longer wavelength transitions become accessible as a result of the type-II band alignment between $\mathrm{GaSb}$ and $\operatorname{InAs} \mathrm{As}_{0.911} \mathrm{Sb}_{0.089}$, but do so at the expense of wavefunction overlap. For a given transition energy in the extended wavelength regime, the design with the optimal wavefunction overlap occurs at the minimum of the wavefunction overlap square contours. The optimal designs are shown by the open circles and provide the best possible wavefunction overlap for a given ground state transition energy of the superlattice.

Another design option at the GaSb lattice constant is the lattice-matched $\mathrm{GaSb} / \mathrm{InAs}_{0.932} \mathrm{Bi}_{0.068}$ superlattice shown in Figs. 8 (a) at $0 \mathrm{~K}$ and 8 (b) at $295 \mathrm{~K}$. Similar to the $\mathrm{GaSb} /$ InAs $_{0.911} \mathrm{Sb}_{0.089}$ superlattice, the $\mathrm{GaSb} / \mathrm{InAs}_{0.932} \mathrm{Bi}_{0.068}$ system also forms a type-II broken gap band alignment, and as a result, the two design spaces bear many similarities. There is a digital alloy regime between the bandgap energies of $\mathrm{GaSb}$ and $\mathrm{InAs}_{0.932} \mathrm{Bi}_{0.068}$, an extended wavelength regime for energies below the bandgap of $\operatorname{In} \mathrm{As}_{0.932} \mathrm{Bi}_{0.068}$, and a nontrivial optimal design in the extended wavelength regime. The key difference between the two superlattice systems is that a greater spectral range falls into the high wavefunction overlap digital alloy regime in the $\mathrm{GaSb} / \mathrm{InAs} \mathrm{s}_{0.932} \mathrm{Bi}_{0.068}$ superlattice due to the significantly smaller bandgap energy of $\operatorname{InAs}_{0.932} \mathrm{Bi}_{0.068}$.

\section{STRAIN-BALANCED InAs/InAsSb, InAs/GalnSb, AND InAs/InAsBi SUPERLATTICES}

In addition to lattice-matched designs, a variety of strain-balanced superlattice systems can be grown at the GaSb lattice constant including InAs/InAsSb, InAs/GaInSb, and InAs/InAsBi. In each of these three material systems, the InAs layers are under tensile strain which is compensated by compressively strained InAsSb, GaInSb, or InAsBi layers. The strain is balanced using the thickness-weighted technique $^{7}$ in which the magnitudes of the strain and thickness product of the tensile and compressive layers are equal. Equation (2) shows the thickness weighted strainbalance expression for an InAs/InAsSb superlattice with layer thicknesses $d_{I n A s}$ and $d_{I n A s S b}$ and strains $\varepsilon_{I n A s}$ and $\varepsilon_{\text {InASSb }}$.

$$
\varepsilon_{I n A s} \times d_{I n A s}+\varepsilon_{I n A s S b} \times d_{I n A s S b}=0 .
$$

The design space map of the strain-balanced InAs/InAsSb superlattice system is presented in Figs. 9(a) at $0 \mathrm{~K}$ and 9(b) at $295 \mathrm{~K}$, where the ground state transition energy of the superlattice is plotted as a function of the InAsSb layer composition for 0 (infinitesimal), 4, 7, 9, 12, and $20 \mathrm{~nm}$ period thicknesses (solid curves). Also shown as a solid curve is a critical thickness limit $^{27}$ which signifies the maximum period thickness that can be utilized before the individual InAs or InAsSb layers begin to relax. Wavefunction overlap square contours are represented with dotted curves which show that a digital alloy regime is formed between the bandgap energies of tensilely strained InAs and lattice-matched InAsSb within which the optimal design follows the infinitesimal $(0 \mathrm{~nm})$ period thickness design contour. Additionally, an extended wavelength regime is formed below the bandgap energy of lattice-
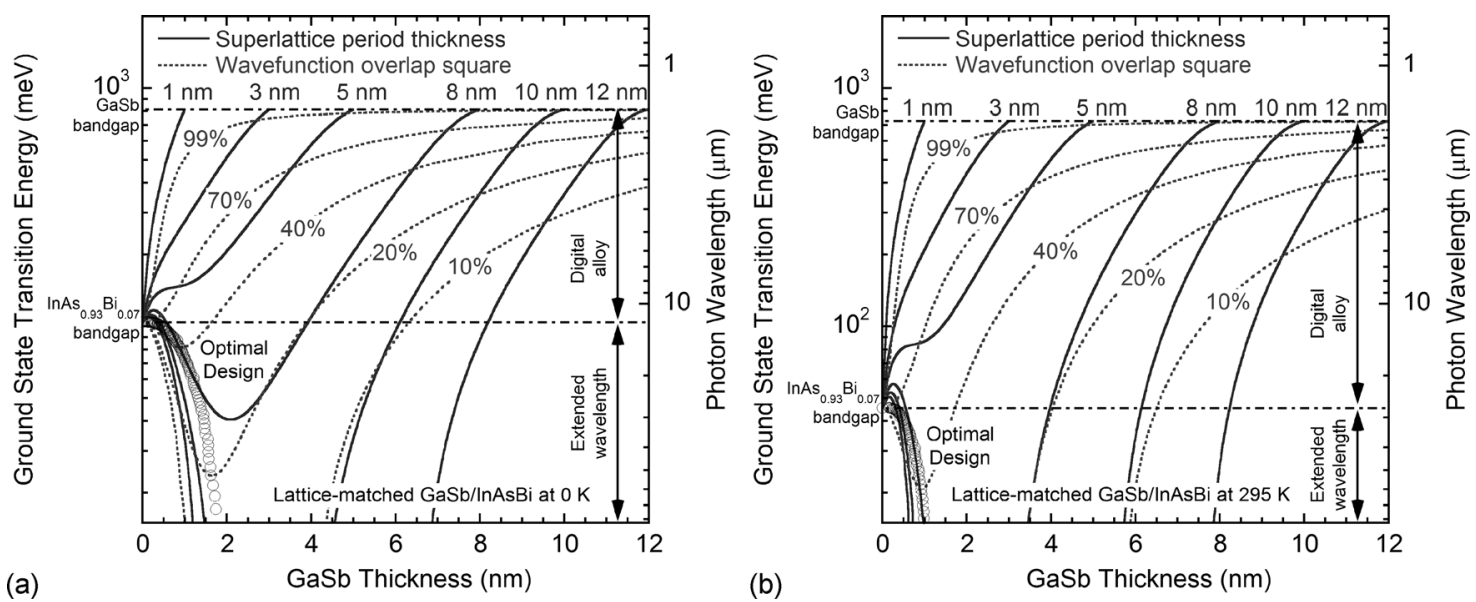

FIG. 8. Ground state transition energy of lattice-matched $\mathrm{GaSb} / \mathrm{InAs}_{0.932} \mathrm{Bi}_{0.068}$ superlattices as a function of GaSb layer thickness; (a) low temperature and (b) room temperature. The solid curves are contours of constant superlattice period. The dotted curves are contours of constant electron-hole wavefunction overlap squared expressed as a percentage. The minima in the wavefunction overlap square contours represent designs that provide maximum absorption for a given transition energy shown by the open circles. 

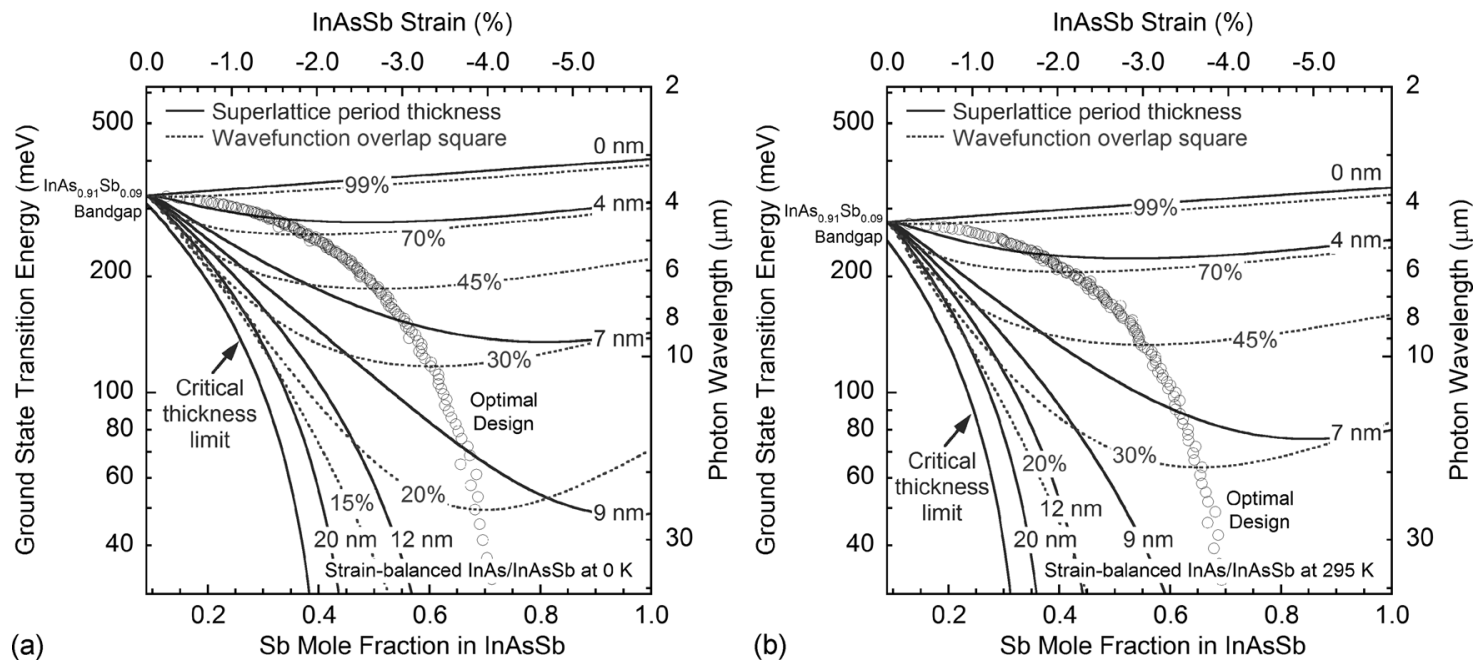

FIG. 9. Ground state transition energy of strain-balanced InAs/InAsSb type-II superlattices as a function of InAsSb mole fraction on the lower horizontal axis and strain on the upper horizontal axis; (a) low temperature and (b) room temperature. The solid curves are contours of constant superlattice period thickness and the critical thickness limit indicates the maximum period thickness that can be grown before the constituent InAs or InAsSb layers begin to relax. The dotted curves are contours of constant electron-hole wavefunction overlap squared expressed as a percentage. The minima in the wavefunction overlap contours represent designs that provide maximum absorption for a given transition energy shown by the open circles.

matched InAsSb within which the optimal design follows the minima of the wavefunction overlap square contours (open circles).

Similar to the digital alloy regimes of the latticematched designs discussed in Section $\mathrm{V}$, the digital alloy regimes of the strain-balanced superlattice designs are bound by the bandgap energies of the superlattice constituents. This particular characteristic of the design is a direct result of the strain-balance condition ${ }^{7}$ given in Equation (2). For example, the lower energy limit of the InAs/InAsSb digital alloy regime coincides with the bandgap energy of $\operatorname{InAs}{ }_{0.911} \mathrm{Sb}_{0.089}$. From the point of view of strain-balance, reducing the $\mathrm{Sb}$ mole fraction (InAsSb strain) produces a corresponding decrease in the ratio of the InAs to InAsSb layer thickness $d_{\text {InAs }} / d_{\text {InAsSb }}$. In the limit that the InAsSb layer strain approaches zero, the InAs layer thickness is zero and the InAsSb layer (lattice-matched $\mathrm{InAs}_{0.911} \mathrm{Sb}_{0.089}$ ) constitutes the full period thickness of the design. The structure of the superlattice becomes independent of period thickness as it converges to bulk lattice-matched $\mathrm{InAs}_{0.911} \mathrm{Sb}_{0.089}$, and the superlattice effective bandgap converges to the bandgap energy of bulk InAs $\mathrm{s}_{0.911} \mathrm{Sb}_{0.089}$; note the convergence of all period thickness contours at the lattice-matched $\mathrm{Sb}$ mole fraction of $8.9 \%$.

Similarly, the upper energy limit of the digital alloy regime approaches the heavy hole bandgap energy of tensilely strained InAs. In this case, increasing the $\mathrm{Sb}$ mole fraction (InAsSb strain) produces a corresponding decrease in the ratio of the InAsSb and InAs layer thicknesses $d_{\text {InAsSb }} / d_{I n A s}$. If the InAsSb layer strain could be made to be arbitrarily large, then the InAsSb layer thickness would approach zero and the superlattice would converge to bulk, tensilely strained InAs on $\mathrm{GaSb}$ for all period thicknesses. In practice, the InAsSb layer strain is limited to $-5.91 \%$ for a unity $\mathrm{Sb}$ mole fraction. As the InAs layer strain is $0.61 \%$, the ratio $d_{\text {InAsSb }} / d_{\text {InAs }}$ can be no smaller than 0.1 ; as a result, the superlattice converges to bulk, tensilely strained InAs on GaSb only for very short period thicknesses.

The next design space considered is that of the strainbalanced InAs/GaInSb superlattice shown in Figs. 10(a) at $0 \mathrm{~K}$ and 10 (b) at $295 \mathrm{~K}$. The ground state transition energy of the superlattice is plotted as a function of the GaInSb layer composition for 0 (infinitesimal), 5, 7, 9, and $10 \mathrm{~nm}$ period thicknesses (solid curves). Critical thickness limited designs produce negative transition energies not seen in Fig. 10 due to the type-II broken gap band alignment between InAs and GaInSb. A digital alloy regime is formed between the GaSb and tensilely strained InAs heavy hole bandgap energies between which the optimal design contour follows the infinitesimal $(0 \mathrm{~nm})$ period thickness. The optimal design in the extended wavelength regime originates at the bandgap energy of tensilely strained InAs and extends to zero and negative (semimetallic) transition energies.

Two important characteristics of the strain-balanced type-II superlattice are observed in Figs. 9 and 10; (i) the digital alloy regimes are bounded by the bandgap energies of the fixed strain binary layers (tensile InAs) and the latticematched compositions of the variable strain alloy layers ( $\mathrm{GaSb}$ and $\left.\mathrm{InAs} \mathrm{As}_{0.911} \mathrm{Sb}_{0.089}\right)$ and (ii) the extended wavelength regime originates at the lower energy end of the digital alloy regime with $100 \%$ wavefunction overlap. This suggests that improved wavefunction overlap could be obtained at longer wavelengths in the extended wavelength regime if a material with a smaller lattice-matched bandgap energy was used in place of InAsSb or GaInSb.

Referring back to Fig. 1, it can be seen that InAsBi possesses several appealing features for superlattice applications. In AsBi layers are compressively strained on $\mathrm{GaSb}$ for Bi mole fractions $>6.8 \%$, its bandgap decreases at a greater rate than either GaInSb or InAsSb, and it forms a type I band alignment with InAs (see Fig. 6 inset). Furthermore, the InAs/InAsBi superlattice is defined solely by the presence of 

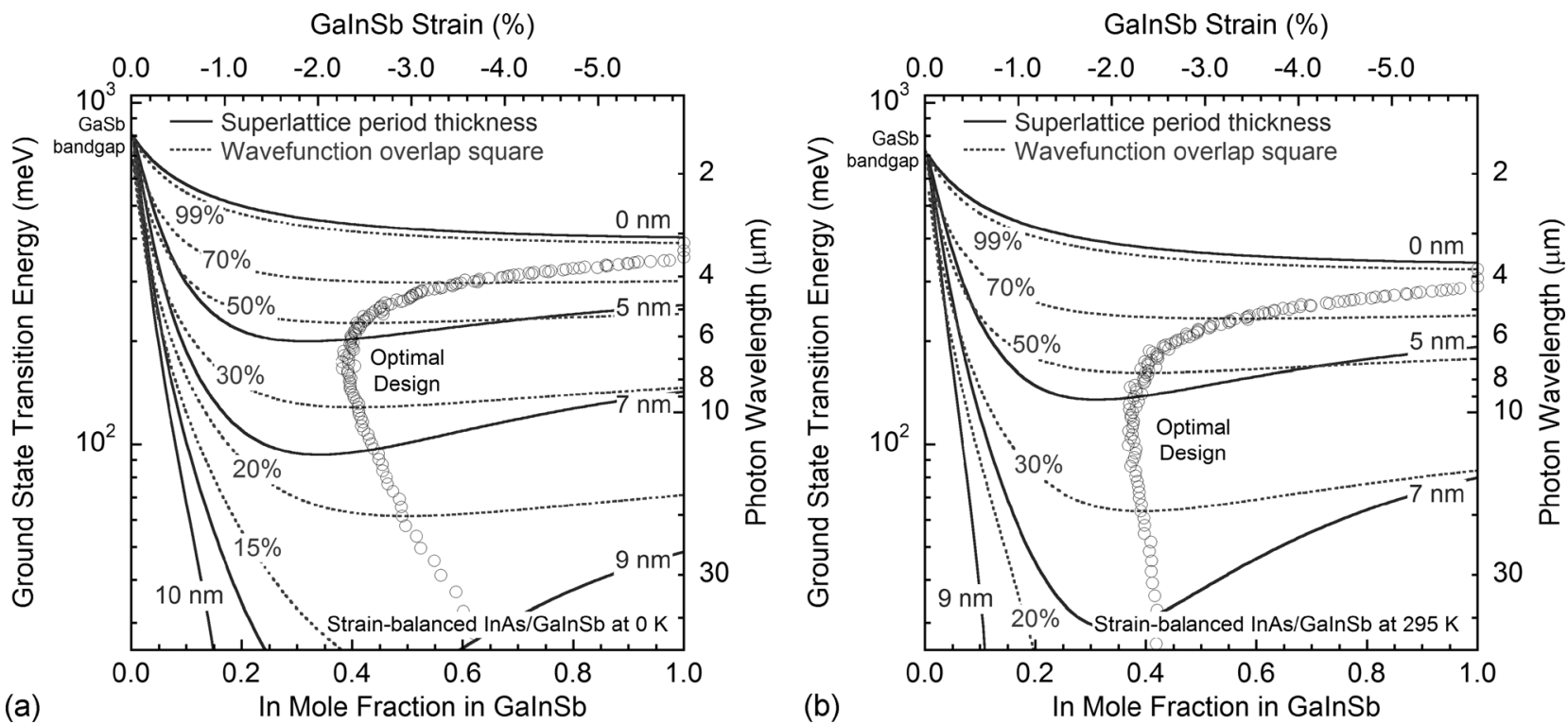

FIG. 10. Ground state transition energy of strain-balanced InAs/GaInSb type-II superlattices as a function of GaInSb mole fraction on the lower horizontal axis and strain on the upper horizontal axis; (a) low temperature and (b) room temperature. The solid curves are contours of constant superlattice period thickness. The dotted curves are contours of constant electron-hole wavefunction overlap squared expressed as a percentage. The minima in the wavefunction overlap contours represent designs that provide maximum absorption for a given transition energy shown by the open circles.

$\mathrm{Bi}$ in the InAsBi layers and the absence of $\mathrm{Bi}$ in the InAs layers, as the group-III sublattice is composed exclusively of In atoms throughout the superlattice. As a result, the InAs/ InAsBi superlattice possesses a relatively simple interface configuration and may therefore be expected to exhibit long minority carrier lifetimes just as in the InAs/InAsSb superlattice. $^{28}$ The design space map of the strain-balanced InAs/ InAsBi superlattice system is presented in Figs. 11(a) at $0 \mathrm{~K}$ and $11(\mathrm{~b})$ at $295 \mathrm{~K}$.

Type-I band alignment between InAs and InAsBi modifies the design space map in Fig. 11 in a few key ways compared to the type-II superlattice design space maps seen in Figs. 9 or 10. Type-I band alignment acts to keep wavefunction overlap relatively high in the extended wavelength regime due to electrons and holes being confined in the same layer (InAsBi). Type-I band alignment also causes the wavefunction overlap square contours to become multivalued functions of $\mathrm{Bi}$ mole fraction, as $100 \%$ wavefunction overlap occurs not just in the infinitesimal $(0 \mathrm{~nm})$ period thickness limit but also in the long period thickness limit where the ground state transition energy approaches the bandgap energy of bulk InAsBi. However, the long period thickness region of the design space is of limited practical use as these designs are essentially just thick, uncoupled InAsBi layers between thick InAs layers which act to balance the strain. In practice, the critical thicknesses of the
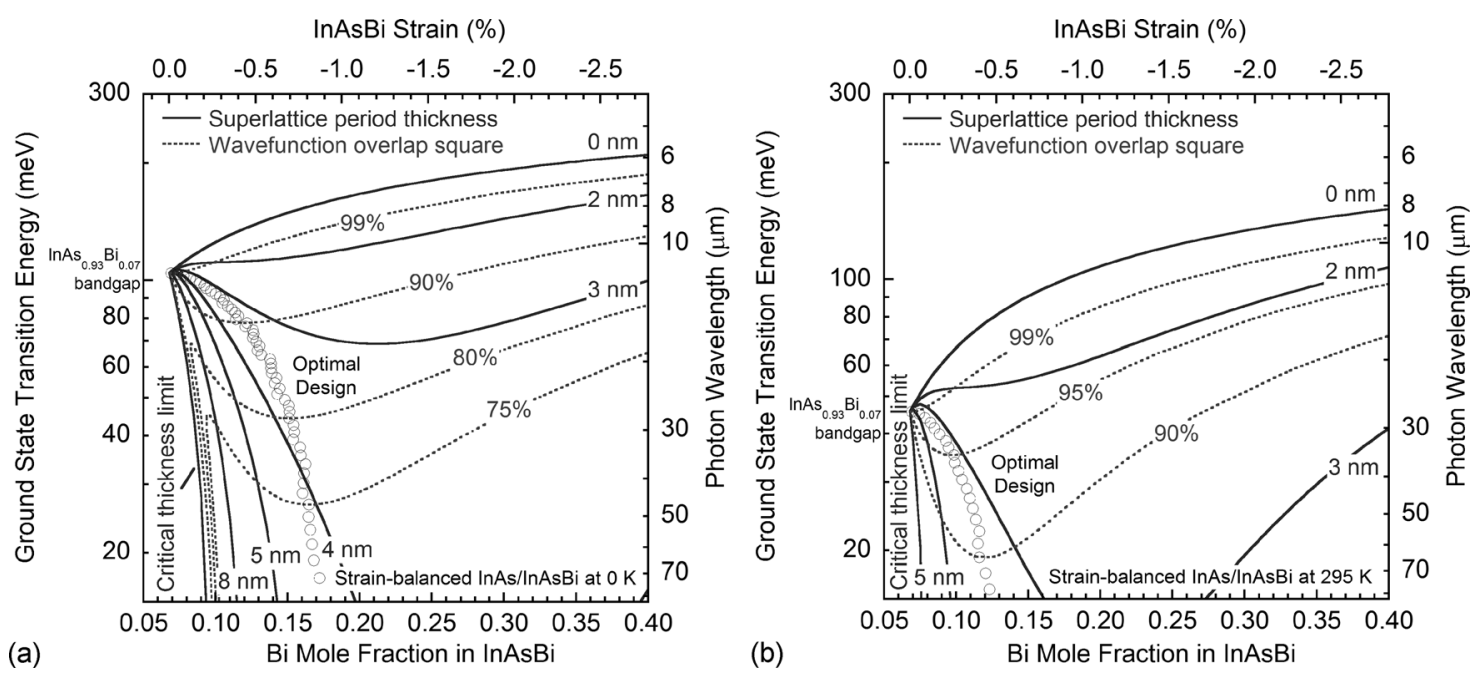

FIG. 11. Ground state transition energy of strain-balanced InAs/InAsBi type-I superlattices as a function of InAsBi mole fraction on the lower horizontal axis and strain on the upper horizontal axis; (a) low temperature and (b) room temperature. The solid curves are contours of constant superlattice period thickness and the critical thickness limit indicates the maximum period thickness that can be grown before the constituent InAs or InAsBi layers begin to relax. The dotted curves are contours of constant electron-hole wavefunction overlap squared expressed as a percentage. The minima in the wavefunction overlap contours represent designs that provide maximum absorption for a given transition energy shown by the open circles. 


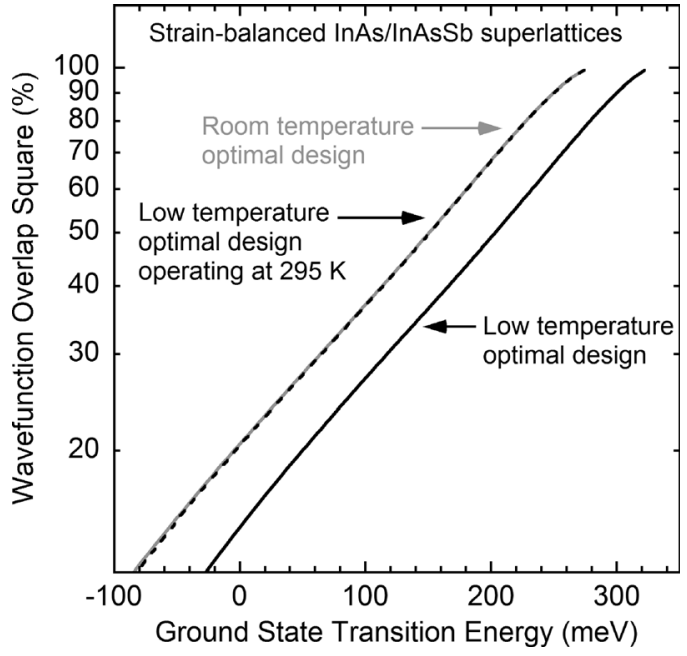

FIG. 12. Wavefunction overlap square plotted as a function of the ground state transition energy of the strain-balanced InAs/InAsSb superlattice. Solid curves represent designs optimized at low temperature (black) and at room temperature (grey). The dashed black curve represents the low temperature optimal design operating at $295 \mathrm{~K}$.

superlattice constituents ultimately limit the longest period thickness designs that can be grown with high epitaxial quality.

\section{TEMPERATURE DEPENDENCE OF THE OPTIMAL DESIGNS}

In this section, the low and room temperature optimal designs are compared to determine whether or not it is necessary to calculate the optimal design parameters as a function of temperature. In Fig. 12, wavefunction overlap square is plotted as a function of ground state transition energy in the extended wavelength regime of the optimal InAs/InAsSb superlattice. The solid black curve represents the low temperature $(0 \mathrm{~K})$ optimal design from Fig. 9(a), and the solid grey curve represents the room temperature $(295 \mathrm{~K})$ optimal design from Fig. 9(b). Next, the transition energies and wavefunction overlap square of the low temperature optimal design are calculated at an operating temperature $295 \mathrm{~K}$ resulting in the dashed black curve which closely follows the room temperature optimal design (grey curve) with virtually no loss in wavefunction overlap.

These results indicate that the optimized InAs/InAsSb superlattice design parameters are not a strong function of temperature; the same conclusion is drawn from the temperature dependent analysis of the optimal InAs/GaInSb, InAs/ InAsBi, GaSb/InAs ${ }_{0.911} \mathrm{Sb}_{0.089}$, and $\mathrm{GaSb} / \mathrm{InAs}_{0.932} \mathrm{Bi}_{0.068}$ superlattice systems. This characteristic of the superlattice can be understood by considering how temperature modifies the bulk band structure of the superlattice constituents. When the temperature is changed, the conduction bands of the two superlattice constituents generally shift in the same direction and often by roughly the same amount. As a result, the repeating potential in the conduction band is virtually unchanged; the electron minibands shift with the bulk band structure, while the wavefunctions are essentially unaltered.
The same is true of the hole minibands formed in the valence band, and thus what is ultimately observed is that the ground state transition energy primarily shifts with the bulk band structure while wavefunction overlap is constant. Therefore, it is only necessary to calculate the optimal design of a superlattice system at a single temperature in order to determine the optimal layer thicknesses and mole fractions as a function of wavefunction overlap. The ground state transition energy of the optimal design is the only parameter that is a strong function of temperature.

\section{SUPERLATTICE ABSORPTION COEFFICIENT}

The ground state absorption coefficient of each superlattice $\alpha_{\mathrm{SL}}$ is computed as a function of the electron-hole wavefunction overlap square $\left|\Psi_{h}^{*} \Psi_{e}\right|^{2}$ using Equation (3). ${ }^{8}$ The proportionality constant, $\alpha_{1}$ in Equation (3), is the ground state absorption coefficient realized in the superlattice when the wavefunction overlap is unity. This constant can be determined experimentally from absorption coefficient measurements and, for example, has been determined as $4990 \mathrm{~cm}^{-1}$ for the strain-balanced InAs/InAsSb superlattice system. ${ }^{8,29} \mathrm{In}$ the InAs/InAsSb superlattice design space (see extended wavelength regime of Fig. 9), the 100\% wavefunction overlap superlattice design converges to bulk $\operatorname{InAs}_{0.911} \mathrm{Sb}_{0.089}$ in the limit that the InAs layer thickness approaches zero and the $\mathrm{Sb}$ mole fraction approaches the lattice-matched condition with unstrained InAsSb constituting the full period thickness.

$$
\alpha_{\mathrm{SL}}=\alpha_{1} \times\left|\Psi_{h}^{*} \Psi_{e}\right|^{2} .
$$

For bulk material, there is only one analytical point in the optical joint density of states at which the bandgap absorption coefficient of the material $\alpha_{g}$ is well defined (see Fig. 4). For a superlattice there are two analytical points, one at the lowest and one at the highest energy transition formed between the ground state electron and hole minibands. The lowest energy transition defines the effective bandgap of the superlattice, whereas at the highest energy transition, the superlattice density of states is constant in photon energy and the absorption coefficient plateaus at $\alpha_{\mathrm{SL}}$ until the onset of the next set of miniband transitions is reached. The maximum of the first derivative of the absorption coefficient identifies the point at which the optical joint density of states increases at the greatest rate and hence provides reliable measure of the superlattice effective bandgap. Nevertheless, the steep rate of increase in the superlattice absorption coefficient at that point makes it difficult to accurately determine the magnitude of the superlattice absorption coefficient at the effective bandgap. Furthermore, since the optical joint density of states and the absorption coefficient plateau at $\alpha_{\mathrm{SL}}$, it can be reliably and reproducibly determined. ${ }^{8}$ There is no bulk material analog for $\alpha_{\mathrm{SL}}$, as it is unique to the superlattice optical joint density of states.

The room temperature absorption coefficient of bulk $\mathrm{InAs}_{0.911} \mathrm{Sb}_{0.089}$ has been measured as a function of photon energy using spectroscopic ellipsometry; ${ }^{20}$ the first derivative maximum of the absorption coefficient identifies the bandgap absorption coefficient $\alpha_{g}$ as $2240 \mathrm{~cm}^{-1}$. The structure of the 
strain-balanced InAs/InAsSb superlattice is convergent on bulk $\operatorname{InAs}_{0.911} \mathrm{Sb}_{0.089}$ in the limit that wavefunction overlap approaches $100 \%$, at which point the absorption coefficient $\alpha_{\mathrm{SL}}=\alpha_{1}=4990 \mathrm{~cm}^{-1}$. The bandgap absorption coefficient $\alpha_{g}$ is smaller than $\alpha_{1}$ as it is measured at a lower density of states. For comparison, the absorption coefficient of $\operatorname{InAs} \mathrm{s}_{0.911} \mathrm{Sb}_{0.089}$ reaches $4990 \mathrm{~cm}^{-1}\left(\alpha_{1}\right)$ when the photon energy is $30 \mathrm{meV}$ greater than the bandgap, which is consistent with the width of the ground state electron miniband in a typical strainbalanced InAs/InAsSb superlattice.

Equation (3) is useful in that it can be employed to determine the absorption coefficient of a given superlattice design from the calculated wavefunction overlap, provided the unity wavefunction overlap absorption coefficient $\alpha_{1}$ is known. To date $\alpha_{1}$ has been measured for strain-balanced InAs/InAsSb superlattices. ${ }^{8}$ Comparison of the measured bandgap absorption coefficient of bulk InAs ${ }_{0.911} \mathrm{Sb}_{0.089} \alpha_{g}=2240 \mathrm{~cm}^{-1}$ and $\alpha_{1}=4990 \mathrm{~cm}^{-1}$ indicates that $\alpha_{1}=C_{\mathrm{SL}} \times \alpha_{g}$ with the constant of proportionality $C_{\mathrm{SL}}=2.228$. Provided this relationship is true in other material systems, the unity wavefunction overlap absorption coefficient $\alpha_{1}$ in Equation (3) can be replaced with the product of $C_{\mathrm{SL}}$ and $\alpha_{g}$ as shown in Equation (4). The significance of this relationship is the bulk bandgap absorption coefficient $\alpha_{g}$ is simpler to determine than $\alpha_{1}$.

$$
\alpha_{\mathrm{SL}}=\left(C_{\mathrm{SL}} \times \alpha_{g}\right) \times\left|\Psi_{h}^{*} \Psi_{e}\right|^{2} .
$$

The bandgap absorption coefficient $\alpha_{g}$ is plotted in Fig. 13 versus the low temperature (solid squares) and room temperature (solid circles) bandgap energy for bulk GaAs, InP, GaSb, InAs, InAs $\mathrm{As}_{0.911} \mathrm{Sb}_{0.089}$, InSb, and InAs $\mathrm{As}_{0.936} \mathrm{Bi}_{0.064}$. The dashed and solid lines are power law equations fit to the respective low and room temperature results, with power law 1.00 observed at low temperature and power law 0.87 at room temperature. The bandgap energies and bandgap

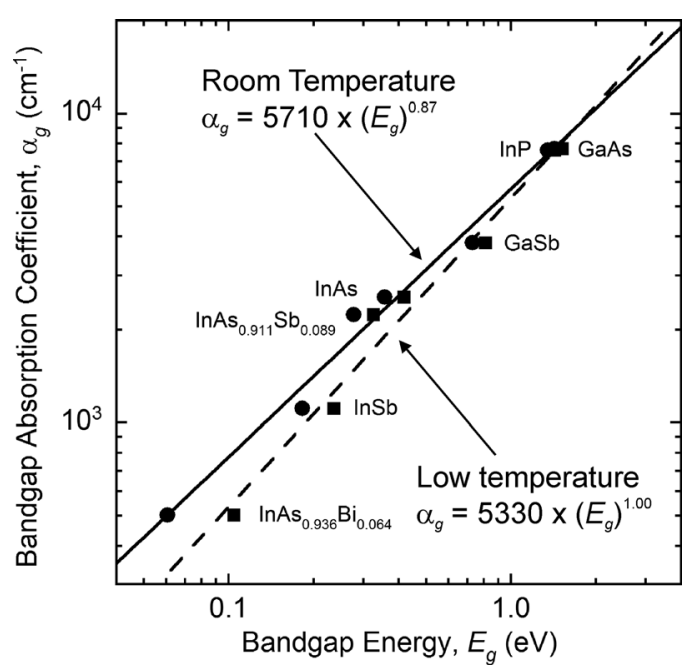

FIG. 13. Bandgap absorption coefficient of various bulk III-V semiconductors as a function of bandgap energy at low temperature (squares) and at room temperature (circles). For all materials, the bandgap energy shifts left to lower energies as the temperature increases. The dashed and solid lines are power law fits to the low and room temperature data, with the best fit equations shown.
TABLE III. Bandgap energy $E_{g}$ and bandgap absorption coefficient $\alpha_{g}$ of bulk III-V binary and ternary alloys determined using spectroscopic ellipsometry.

\begin{tabular}{lccc}
\hline \hline Material & $E_{g}$ at $295 \mathrm{~K}(\mathrm{eV})$ & $E_{g}$ at $0 \mathrm{~K}(\mathrm{eV})$ & $\alpha_{g}\left(\mathrm{~cm}^{-1}\right)$ \\
\hline $\mathrm{GaAs}$ & 1.425 & 1.519 & 7700 \\
$\mathrm{InP}$ & 1.348 & 1.424 & 7615 \\
$\mathrm{GaSb}$ & 0.730 & 0.812 & 3820 \\
$\mathrm{InAs}$ & 0.357 & 0.417 & 2550 \\
$\mathrm{InSb}$ & 0.182 & 0.235 & 1112 \\
$\mathrm{InAs}$ & 0.277 & 0.325 & 2240 \\
$\mathrm{InAs}_{0.936} \mathrm{Sb}_{0.089}$ & 0.061 & 0.104 & 503 \\
\hline \hline
\end{tabular}

absorption coefficients are summarized in Table III. In these materials, the functional form and magnitude of the optical joint density of states is insensitive to temperature, while the accompanying bandgap energy is strongly temperature dependent.

The bandgap absorption coefficient $\alpha_{g}$ and room temperature bandgap energy are obtained from spectroscopic ellipsometry measurements and analysis of each material absorption coefficient as a function of energy. In the analyses, the bandgap absorption coefficient and energy are determined using the first derivative maximum method. ${ }^{20}$ The low temperature bandgap energies are obtained from the literature for the binary materials ${ }^{2}$ and $\mathrm{InAs}_{0.911} \mathrm{Sb}_{0.089},{ }^{20}$ and from this work for $\operatorname{InAs}_{0.932} \mathrm{Bi}_{0.064}$.

These results show that the bandgap absorption coefficient significantly decreases with bandgap energy, which is primarily due to a strong reduction in the optical joint density of states in small bandgap III-Vs. On the other hand, superlattices maintain a relatively constant optical joint density of states within a given set of miniband transitions and the reduction observed in the superlattice bandgap absorption coefficient with effective bandgap energy is mainly due to a reduction in the wavefunction overlap square. $^{8}$

\section{COMPARING ABSORPTION AND PRACTICAL DESIGN CONSIDERATIONS}

The absorption coefficients of the superlattice systems discussed in Sections V and VI are calculated as a function of wavefunction overlap square using Equation (4), the constant of proportionality $C_{\mathrm{SL}}=2.228$, and the bandgap absorption coefficients $\alpha_{g}$ listed in Table III. The 100\% wavefunction overlap designs for lattice-matched $\mathrm{GaSb} / \mathrm{InAs}_{0.911} \mathrm{Sb}_{0.089}$ and strain-balanced InAs/InAsSb superlattices both converge to bulk InAs ${ }_{0.911} \mathrm{Sb}_{0.089}$ (see Figs. 7 and 9), and thus the measured value of $\alpha_{1}=C_{\mathrm{SL}} \times \alpha_{g}=4990 \mathrm{~cm}^{-1}$ for InAs/InAsSb superlattices is used. For lattice-matched $\mathrm{GaSb} / \mathrm{InAs} \mathrm{As}_{0.936} \mathrm{Bi}_{0.068}$ and strain-balanced InAs/InAsBi superlattices, the bandgap absorption coefficient for bulk $\operatorname{InAs}{ }_{0.936} \mathrm{Bi}_{0.064}$ is used with $\alpha_{1}=C_{\mathrm{SL}} \times \alpha_{g}=1120 \mathrm{~cm}^{-1}$. Likewise for strain-balanced InAs/GaInSb superlattices, the bandgap absorption coefficient for bulk InAs is utilized with $\alpha_{1}=C_{\mathrm{SL}} \times \alpha_{g}=5680 \mathrm{~cm}^{-1}$. In the digital alloy regimes, the absorption coefficients are calculated by interpolating $\alpha_{1}$ as a function of energy between the 


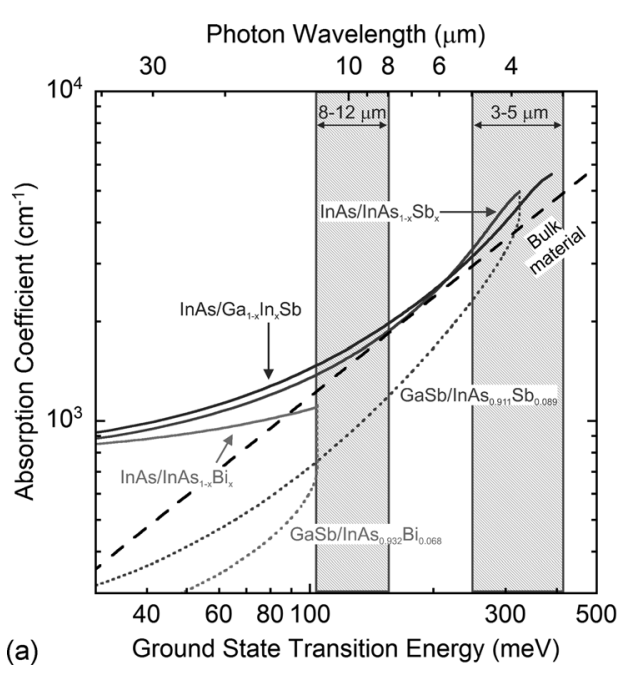

(a)

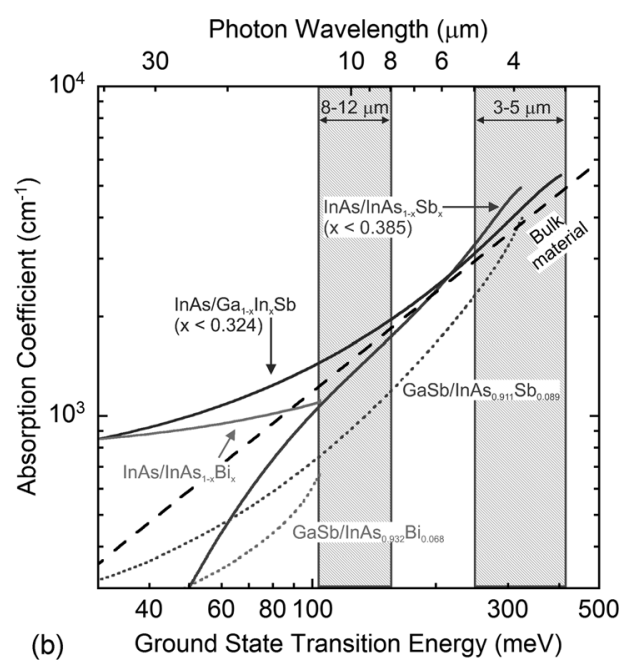

FIG. 14. Absorption coefficient of optimized superlattice designs as a function of low temperature ground state transition energy on the lower horizontal axis and corresponding photon wavelength cutoff on the upper horizontal axis; (a) optimal designs and (b) strain- and thickness-limited practical designs. Strain-balanced designs are shown by solid curves, lattice-matched designs are shown by dotted curves, and bulk material is shown by a dashed line. The technologically relevant 3 to 5 and 8 to $12 \mu \mathrm{m}$ wavelength ranges are indicated by vertical shaded bands. two endpoint materials of the digital alloy, and scaling the resultant absorption coefficient by the square of the electron-hole wavefunction overlap.

The absorption coefficients of the strongest possible absorbing superlattice designs in the extended wavelength regime are provided in Fig. 14 as a function of the low temperature ground state transition energy (lower horizontal axis) and the corresponding wavelength cutoff (upper horizontal axis). The overall optimal designs are shown in Fig. 14(a), and the practical designs limited by achievable material strain and thicknesses are shown in Fig. 14(b). The three strain-balanced systems (InAs/InAsSb, InAs/GaInSb, and InAs/InAsBi) are shown by solid curves, and the two lattice-matched systems $\left(\mathrm{GaSb} / \mathrm{In} \mathrm{As}_{0.911} \mathrm{Sb}_{0.089}\right.$ and $\left.\mathrm{GaSb} / \mathrm{InAs}_{0.936} \mathrm{Bi}_{0.068}\right)$ are shown by dotted curves. To compare the absorption strength of the optimal superlattice designs with that of bulk material, the power law fit for low temperature bulk material shown in Fig. 13 is scaled by the proportionality constant $C_{\mathrm{SL}}$ and displayed by the dashed line; the resulting equation is 2.228 $\times 5.330 \mathrm{~cm}^{-1} / \mathrm{meV} \times E_{g}$, where $E_{g}$ is the ground state transition energy in units of meV.

For the InAsSb- and InAsBi-based superlattices, the strain-balanced systems provide stronger absorption as a function of transition energy than their lattice-matched counterparts. Physically, this occurs because the strainbalanced systems utilize the ternary layer mole fraction as a free design parameter which allows more favorable band alignments to be established. For all five superlattice systems, the absorption coefficient decreases as the wavefunction overlap becomes weaker with increasing wavelength cutoff, and the rate at which the absorption coefficient decreases is lowest for the strain-balanced InAs/InAsBi superlattice due to its type-I band alignment. In fact for very long wavelengths $(>12 \mu \mathrm{m})$, the rate at which the strain-balanced superlattice absorption coefficients decrease with photon energy due to decreasing wavefunction overlap (solid curves) is significantly less than the rate at which the bulk absorption coefficient decreases (dashed line), indicating that superlattice-based designs may be preferable to bulk material in terms of absorption strength for longer wavelength applications.
While the optimal designs discussed thus far are useful as a design guide, the practical design must abide by additional constraints inherent to epitaxial growth. For example, the magnitude of the strain on the constituent layers becomes a practical design concern as the optical quality degrades rapidly when the material is heavily strained, even if the layer is pseudomorphic. Fig. 14(b) shows the practical design curves in which the magnitude of the ternary layer strain in the strain-balanced superlattice systems is limited to $2.0 \%$, which limits the $\mathrm{Sb}$ mole fraction to 0.385 in InAsSb and the In mole fraction to 0.324 in GaInSb. With this added constraint, the strain-balanced InAs/InAsSb superlattice is most strongly influenced; however, it still outperforms its lattice-matched counterpart $\mathrm{GaSb} / \mathrm{InAs}_{0.911} \mathrm{Sb}_{0.089}$. On the other hand, the strain-balanced InAs/GaInSb superlattice is influenced less because the wavefunction overlap square contours in this system are relatively flat in the vicinity of the optimal design (see Fig. 10) resulting in an optimal design that is relatively insensitive to In-content. Nevertheless, the strain-balanced InAs/ InAsSb superlattice offers a significantly longer minority carrier lifetime compared to the strain-balanced InAs/GaInSb superlattice. ${ }^{28}$ Absorption in the strain-balanced InAs/InAsBi superlattice is not impacted by the $-2 \%$ strain limit as the InAsBi strain in the optimal design never exceeds $-1 \%$.

While none of the optimal designs investigated exceed the critical thickness limit which would effectively limit the maximum layer thicknesses that could be utilized, several designs do converge upon the infinitesimal $(0 \mathrm{~nm})$ period thickness limit. In addition to the strain limit, the practical design also enforces a minimum layer thickness of $0.50 \mathrm{~nm}$ as a practical limit of how thin these layers could be grown. This constraint mainly appears in the digital alloy regimes of the superlattice designs investigated; however, it also limits the utility of the two lattice-matched systems which require $\mathrm{GaSb}$ thicknesses $<0.50 \mathrm{~nm}$ to reach near-unity wavefunction overlap. The practical and optimal design parameters for the lattice-matched $\mathrm{GaSb} / \mathrm{InAs}_{0.911} \mathrm{Sb}_{0.089}$ and $\mathrm{GaSb} / \mathrm{InAs} \mathrm{As}_{0.932} \mathrm{Bi}_{0.068}$ superlattices and strain-balanced InAs/InAsSb, InAs/GaInSb, and InAs/InAsBi superlattice systems are presented for several key wavelengths in Appendix B. 


\section{VERIFICATION OF THE InAs/InAsSb MID-WAVE OPTIMAL DESIGN}

The optimal design criteria in Appendix B are utilized in the design and growth of an optimized mid-wave infrared strain-balanced InAs/InAsSb superlattice. The sample is composed of alternating InAs $(2.16 \mathrm{~nm})$ and $\operatorname{InAs} \mathrm{s}_{0.802} \mathrm{Sb}_{0.198}$ $(1.51 \mathrm{~nm})$ layers with a total thickness of $1 \mu \mathrm{m}$ sandwiched between $10 \mathrm{~nm}$ thick AlSb confinement layers with a $10 \mathrm{~nm}$ thick GaSb cap. The sample is grown at $425^{\circ} \mathrm{C}$ on a (100)oriented $\mathrm{GaSb}$ substrate by molecular beam epitaxy using a 0.5 monolayer per second InAs growth rate and a constant $1.20 \mathrm{As} / \mathrm{In}$ flux ratio. The structural properties of the superlattice are measured using X-ray diffraction. The unintentional $\mathrm{Sb}$ in the InAs layers of the superlattice is not quantifiable due to the short period thickness of this sample; therefore, an unintentional $\mathrm{Sb}$ mole fraction of 0.024 is assumed based on the unintentional $\mathrm{Sb}$ measured in other InAs/InAsSb superlattices grown under the similar conditions. ${ }^{20}$ The sample cross section is shown in the inset of Fig. 15.

The software tool (see Section IV) utilized to calculate the miniband structure of the superlattice indicates that the ground state transition energy of the superlattice is $305.9 \mathrm{meV}(4.1 \mu \mathrm{m}$ wavelength) at low temperature and $258.1 \mathrm{meV}$ (4.8 $\mu \mathrm{m}$ wavelength) at room temperature, and the square of the electron-hole wavefunction overlap at the ground state is $94.3 \%$. The room temperature absorption coefficient of the superlattice measured by spectroscopic ellipsometry is shown in Fig. 15. The ground state transition energy (effective bandgap) of the superlattice is identified at the absorption coefficient first derivative maximum at $257 \mathrm{meV}$, at which point the absorption coefficient increases rapidly to a value of $4750 \mathrm{~cm}^{-1}$.

The absorption coefficient $\left(\alpha_{\mathrm{SL}}\right)$ of strain-balanced InAs/ InAsSb superlattices as a function of the wavefunction overlap square is shown in Fig. 16. The data represented by open

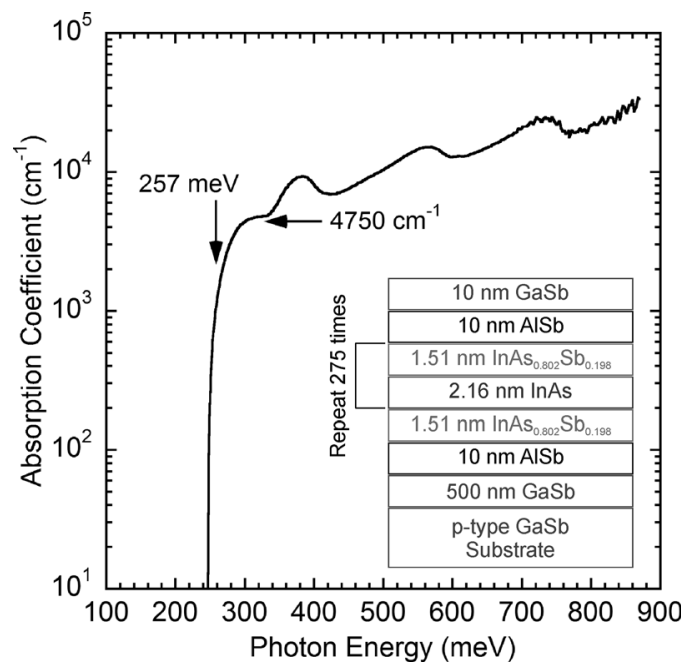

FIG. 15. Room temperature absorption coefficient of a strain-balanced InAs/ InAsSb superlattice optimized for maximum absorption at a ground state transition energy of $257 \mathrm{meV}(4.8 \mu \mathrm{m}$ wavelength). Inset shows the sample cross section.

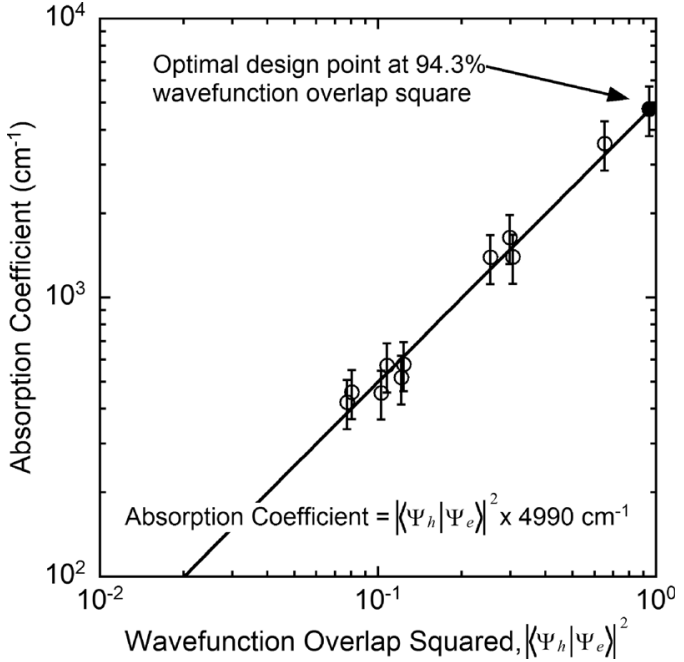

FIG. 16. Ground state absorption coefficient of strain-balanced InAs/InAsSb superlattices as a function of the square of the electron-hole wavefunction overlap. The solid line shows a unity power law fit to the data from Ref. 8 (open circles). The solid circle shows the optimized mid-wave infrared InAs/InAsSb superlattice result with $94.3 \%$ wavefunction overlap square.

circles are taken from Ref. 8 with the wavefunction overlap values updated to account for the unintentional incorporation of $\mathrm{Sb}$ in the InAs layers of the superlattices. ${ }^{20}$ These data yield the unity power law fit (solid line) with the $100 \%$ wavefunction overlap absorption coefficient of $4990 \mathrm{~cm}^{-1}$ that is utilized in Sections VIII and IX. The solid circle shows the absorption coefficient from the optimized InAs/ InAsSb superlattice design (Fig. 15 inset) with 94.3\% wavefunction overlap square, which is in good agreement with that predicted by Equation (4).

A Nicolet Instrument Corporation Magna-IR 760 Fourier transform infrared spectrometer is used to measure room temperature photoluminescence from the $1.0 \mu \mathrm{m}$ thick optimal InAs/InAsSb superlattice grown at $425^{\circ} \mathrm{C}$ as well as a $0.5 \mu \mathrm{m}$ thick bulk lattice-matched $\operatorname{InAs}_{0.911} \mathrm{Sb}_{0.089}$ sample grown at $430{ }^{\circ} \mathrm{C}^{20}$ The samples are excited using an $808 \mathrm{~nm}$ pump laser and $200 \mathrm{~mW}$ of laser power. The pump power density reaching the active region of each sample is $265 \mathrm{~W} \mathrm{~cm}^{-2}$ which corresponds to an excitation density of $1 \times 10^{25} \mathrm{~cm}^{-3} \mathrm{~s}^{-1}$ in the superlattice and $2 \times 10^{25} \mathrm{~cm}^{-3} \mathrm{~s}^{-1}$ in the bulk InAs $\mathrm{As}_{0.911} \mathrm{Sb}_{0.089}$ sample. The raw photoluminescence from the superlattice (dotted black curve) and the bulk In $\mathrm{As}_{0.911} \mathrm{Sb}_{0.089}$ layer (dotted grey curve) are plotted as a function of photon energy (lower horizontal axis) and photon wavelength (upper horizontal axis) in Fig. 17. The raw photoluminescence spectra exhibit an absorption feature near $290 \mathrm{meV}$ due to the presence of $\mathrm{CO}_{2}$ in the air which is corrected in the solid black and grey curves by scaling the spectra according to the optical throughput of the system.

The photoluminescence first derivative maximum is used to identify the room temperature bandgap energy of the bulk $\mathrm{InAs}_{0.911} \mathrm{Sb}_{0.089}$ sample as $275 \mathrm{meV}$, which is in agreement with a room temperature bandgap energy of $277 \mathrm{meV}$ measured using spectroscopic ellipsometry. ${ }^{20}$ Using the same analysis on the superlattice, the photoluminescence first derivative maximum identifies an effective bandgap 


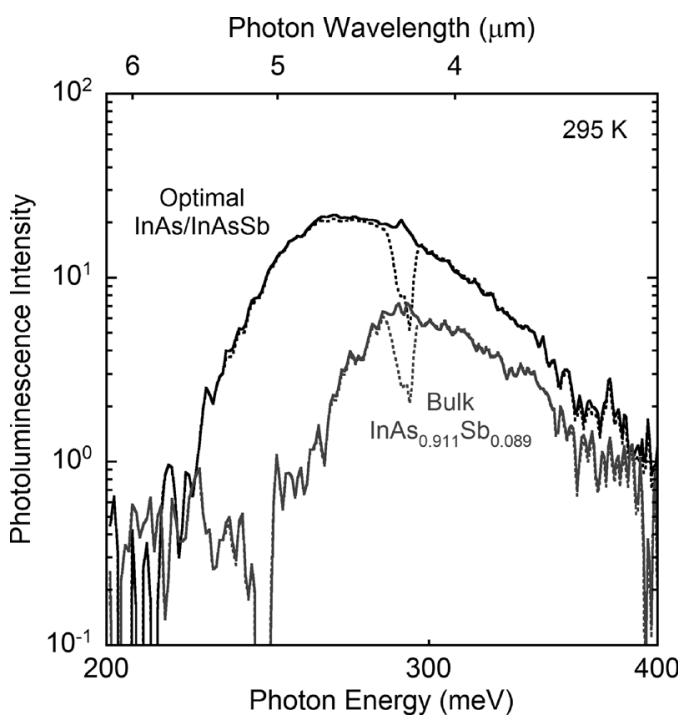

FIG. 17. Room temperature photoluminescence from bulk lattice-matched $\mathrm{InAs}_{0.911} \mathrm{Sb}_{0.089}$ (grey curves) and an InAs/InAsSb superlattice designed to provide optimal wavefunction overlap square at $4.8 \mu \mathrm{m}$ wavelength (black curves). Dotted curves show the raw photoluminescence spectra while solid curves show the spectra after correcting for the optical throughput of the system.

$249 \mathrm{meV}$, which likewise agrees with the $257 \mathrm{meV}$ bandgap measured using spectroscopic ellipsometry in Fig. 15. By integrating the photoluminescence spectra as a function of photon energy, a relative measure of the total optical output and general optical quality is obtained. The total integrated intensity from the optimized superlattice is more than a factor of three greater than the integrated intensity from the bulk $\operatorname{InAs}_{0.911} \mathrm{Sb}_{0.089}$ sample. The superior optical quality of the superlattice is attributed to the high wavefunction overlap of the optimized design and the long minority carrier lifetimes inherent to this material system. ${ }^{28}$

\section{CONCLUSIONS}

The valance band anticrossing model is fit to room temperature measurements of the bulk bandgap energies of $\mathrm{InAs}_{0.936} \mathrm{Bi}_{0.064}(60.6 \mathrm{meV})$ and InAs $(354.0 \mathrm{meV})$ to experimentally determine the $\mathrm{Bi}$ coupling parameter $(1.529 \mathrm{eV})$ for the InAsBi material system. A software tool is developed that determines the miniband structure of semiconductor superlattices and identifies the optimal strain-balanced and latticematched designs for maximum wavefunction overlap. The best superlattice design parameters are provided for latticematched $\mathrm{GaSb} / \mathrm{InAs}_{0.911} \mathrm{Sb}_{0.089}$ and $\mathrm{GaSb} / \mathrm{InAs}_{0.932} \mathrm{Bi}_{0.068}$ and strain-balanced InAs/InAsSb, InAs/GaInSb, and InAs/InAsBi on $\mathrm{GaSb}$. The expected ground state absorption coefficients for these designs are provided as a function of ground state transition energy. The strain-balanced InAs/InAsSb superlattice best design with a low temperature $4.1 \mu \mathrm{m}(306 \mathrm{meV})$ wavelength cutoff $(4.8 \mu \mathrm{m}$ at room temperature) is grown, measured, and provided the ground state absorption coefficient predicted by the model.

\section{ACKNOWLEDGMENTS}

The authors acknowledge financial support through the National Science Foundation (DMR-1410393) and the U.S. Army Research Office MURI program, Grant No. W911NF10-1-0524, as well as the use of facilities in the LeRoy Eyring Center for Solid State Science at Arizona State University.

\section{APPENDIX A: MATERIAL PARAMETERS FOR SUPERLATTICE DESIGN CALCULATIONS}

Table of structural and band structure parameters utilized in the design optimization calculations.

\begin{tabular}{|c|c|c|c|}
\hline Parameter & $\mathrm{Ga}_{1-x} \mathrm{In}_{x} \mathrm{Sb}$ & $\operatorname{InAs}_{1-x} \mathrm{Sb}_{x}$ & $\operatorname{InAs}_{1-x} \mathrm{Bi}_{x}$ \\
\hline $\begin{array}{l}0 \mathrm{~K} \text { unstrained conduction } \\
\text { band edge }(\mathrm{meV})\end{array}$ & $x \cdot(235)+(1-x)(782)-x \cdot(1-x)(413)$ & $x \cdot(235)+(1-x)(-173)-x \cdot(1-x)(558)$ & $x \cdot(-1411)+(1-x)(-173)$ \\
\hline $\begin{array}{l}295 \mathrm{~K} \text { unstrained conduction } \\
\text { band edge }(\mathrm{meV})\end{array}$ & $x \cdot(172)+(1-x)(699)-x \cdot(1-x)(398)$ & $x \cdot(172)+(1-x)(-236)-x \cdot(1-x)(383)$ & $x \cdot(-1411)+(1-x)(-236)$ \\
\hline $\begin{array}{l}0 \mathrm{~K} \text { unstrained valence } \\
\text { band edge }(\mathrm{meV})\end{array}$ & $(1-x)(-30)$ & $(1-x)(-590)-x \cdot(1-x)(-380)$ & $\begin{array}{l}\text { Band anticrossing model: } \\
\text { See Equation (1) and Table I }\end{array}$ \\
\hline $\begin{array}{l}295 \mathrm{~K} \text { unstrained valence } \\
\text { band edge (meV) }\end{array}$ & $(1-x)(-30)$ & $(1-x)(-590)-x \cdot(1-x)(-367)$ & $\begin{array}{l}\text { Band anticrossing model: } \\
\text { See Equation (1) and Table I }\end{array}$ \\
\hline Electron effective mass & $\begin{array}{c}x \cdot(0.0135)+(1-x)(0.0400) \\
-x \cdot(1-x)(0.0092)\end{array}$ & $\begin{array}{c}x \cdot(0.0135)+(1-x)(0.0260) \\
-x \cdot(1-x)(0.0350)\end{array}$ & 0.0260 \\
\hline Heavy hole effective mass & $x \cdot(0.2632)+(1-x)(0.2500)$ & $x \cdot(0.2632)+(1-x)(0.3333)$ & 0.3333 \\
\hline Light hole effective mass & $x \cdot(0.0152)+(1-x)(0.0439)-x \cdot(1-x)(0.0110)$ & $x \cdot(0.0152)+(1-x)(0.0270)$ & 0.0270 \\
\hline Lattice constant (nm) & $x \cdot(0.64792)+(1-x)(0.60957)$ & $x \cdot(0.64792)+(1-x)(0.60582)$ & $x \cdot(0.66108)+(1-x)(0.60582)$ \\
\hline Poisson's ratio & $x \cdot(0.3530)+(1-x)(0.3129)$ & $x \cdot(0.3530)+(1-x)(0.3521)$ & $x \cdot(0.3503)+(1-x)(0.3521)$ \\
\hline $\begin{array}{l}\text { Conduction band deformation } \\
\text { potential }(\mathrm{eV})\end{array}$ & $x \cdot(-6.94)+(1-x)(-7.50)$ & $x \cdot(-6.94)+(1-x)(-5.08)$ & -5.08 \\
\hline $\begin{array}{l}\text { Valence band deformation } \\
\text { potential }(\mathrm{eV})\end{array}$ & $x \cdot(-0.36)+(1-x)(-0.80)$ & $x \cdot(-0.36)+(1-x)(-1.00)$ & -1.00 \\
\hline $\begin{array}{l}\text { Shear deformation } \\
\text { potential }(\mathrm{eV})\end{array}$ & -2.00 & $x \cdot(-2.00)+(1-x)(-1.80)$ & -1.80 \\
\hline
\end{tabular}




\section{APPENDIX B: SUPERLATTICE OPTIMAL DESIGN CRITERIA}

Design parameters for the lattice-matched $\mathrm{GaSb} / \mathrm{InAs}_{0.911} \mathrm{Sb}_{0.089}$ and $\mathrm{GaSb} / \mathrm{InAs}_{0.932} \mathrm{Bi}_{0.068}$ superlattices. Electron-hole wavefunction overlap square $\left|\Psi_{h}^{*} \Psi_{e}\right|^{2}$, absorption coefficient $\alpha_{\mathrm{SL}}$, and the superlattice constituent layer thicknesses are provided for several key detector wavelengths at low temperature $(0 \mathrm{~K})$ and at room temperature $(295 \mathrm{~K})$. When the optimal design calls for thicknesses less than $0.500 \mathrm{~nm}$, an alternate set of practical design parameters is also provided which limit the minimum layer thickness to $0.500 \mathrm{~nm}$. The horizontal dashed line separates designs in the digital alloy regime (above) and extended wavelength regime (below). In the digital alloy regime, the optimal design calls for an infinitesimal period thickness superlattice; therefore, only the practical design parameters are given.

\begin{tabular}{|c|c|c|c|c|c|c|}
\hline \multicolumn{2}{|c|}{ Detector wavelength $(\mu \mathrm{m})$} & \multirow{2}{*}{$\begin{array}{l}\text { Wavefunction } \\
\text { overlap square } \\
\left|\Psi_{h}^{*} \Psi_{e}\right|^{2}(\%)\end{array}$} & \multirow{2}{*}{$\begin{array}{l}\text { Absorption } \\
\text { coefficient } \\
\alpha_{\mathrm{SL}}\left(\mathrm{cm}^{-1}\right)\end{array}$} & \multirow{2}{*}{$\begin{array}{c}\text { GaSb } \\
\text { thickness (nm) }\end{array}$} & \multirow{2}{*}{$\begin{array}{c}\mathrm{InAs}_{0.911} \mathrm{Sb}_{0.089} \\
\text { thickness (nm) }\end{array}$} & \multirow{2}{*}{$\begin{array}{c}\text { Optimal } \\
\text { or practical }\end{array}$} \\
\hline at $0 \mathrm{~K}$ & at $295 \mathrm{~K}$ & & & & & \\
\hline \multicolumn{7}{|c|}{ Lattice-matched $\mathrm{GaSb} / \mathrm{InAs}_{0.911} \mathrm{Sb}_{0.089}$} \\
\hline 3.0 & 3.5 & 97.4 & 5484 & 0.500 & 1.540 & Practical \\
\hline 4.0 & 4.8 & 71.2 & 3553 & 0.550 & 4.630 & Optimal \\
\hline 5.0 & 6.4 & 46.3 & 2310 & 1.170 & 4.350 & Optimal \\
\hline 8.0 & 12.1 & 23.8 & 1188 & 1.870 & 5.650 & Optimal \\
\hline 10.0 & 17.2 & 18.2 & 908 & 2.050 & 6.380 & Optimal \\
\hline 12.0 & 23.9 & 15.0 & 749 & 2.210 & 6.880 & Optimal \\
\hline 15.0 & 39.1 & 12.2 & 609 & 2.400 & 7.400 & Optimal \\
\hline \multicolumn{2}{|c|}{ Detector wavelength $(\mu \mathrm{m})$} & Wavefunction & Absorption & & & \\
\hline at $0 \mathrm{~K}$ & at $295 \mathrm{~K}$ & $\begin{array}{c}\text { overlap square } \\
\left|\Psi_{h}^{*} \Psi_{e}\right|^{2}(\%)\end{array}$ & $\begin{array}{l}\text { coefficient } \\
\alpha_{\mathrm{SL}}\left(\mathrm{cm}^{-1}\right)\end{array}$ & $\begin{array}{c}\text { GaSb } \\
\text { thickness (nm) }\end{array}$ & $\begin{array}{c}\mathrm{InAs}_{0.932} \mathrm{Bi}_{0.068} \\
\text { thickness }(\mathrm{nm})\end{array}$ & $\begin{array}{c}\text { Optimal } \\
\text { or practical }\end{array}$ \\
\hline \multicolumn{7}{|c|}{ Lattice-matched $\mathrm{GaSb} / \mathrm{InAs}_{0.932} \mathrm{Bi}_{0.068}$} \\
\hline 3.0 & 3.6 & 99.8 & 4334 & 0.500 & 0.610 & Practical \\
\hline 4.0 & 5.1 & 99.3 & 3240 & 0.500 & 1.090 & Practical \\
\hline 5.0 & 6.7 & 98.3 & 2571 & 0.500 & 1.640 & Practical \\
\hline 8.0 & 13.2 & 90.0 & 1479 & 0.500 & 3.830 & Practical \\
\hline 10.0 & 19.4 & 76.8 & 1013 & 0.500 & 5.810 & Practical \\
\hline 12.0 & 28.0 & 60.0 & 667 & 0.280 & 13.900 & Optimal \\
\hline 12.0 & 28.4 & 58.2 & 647 & 0.500 & 8.300 & Practical \\
\hline 15.0 & 55.1 & 40.7 & 453 & 0.860 & 7.160 & Optimal \\
\hline
\end{tabular}

Design parameters for the strain-balanced InAs/InAsSb, InAs/GaInSb, and InAs/InAsBi superlattices. Electron-hole wavefunction overlap square $\left|\Psi_{h}^{*} \Psi_{e}\right|^{2}$, absorption coefficient $\alpha_{\mathrm{SL}}$, and the superlattice constituent layer thicknesses and mole fractions are provided for several key detector wavelengths at low temperature $(0 \mathrm{~K})$ and at room temperature $(295 \mathrm{~K})$. When the optimal design calls for thicknesses less than $0.500 \mathrm{~nm}$ or strain exceeding $\pm 2 \%$, an alternate set of practical design parameters is also provided which limit the minimum layer thickness to $0.500 \mathrm{~nm}$ and the maximum strain to $\pm 2 \%$. The horizontal dashed line separates designs in the digital alloy regime (above) and extended wavelength regime (below). In the digital alloy regime, the optimal design calls for an infinitesimal period thickness superlattice; therefore, only the practical design parameters are given.

Strain-balanced InAs/InAsSb.

\begin{tabular}{|c|c|c|c|c|c|c|c|}
\hline \multicolumn{2}{|c|}{ Detector wavelength $(\mu \mathrm{m})$} & \multirow{2}{*}{$\begin{array}{c}\text { Wavefunction } \\
\text { overlap square } \\
\left|\Psi_{h}^{*} \Psi_{e}\right|^{2}(\%)\end{array}$} & \multirow{2}{*}{$\begin{array}{c}\text { Absorption } \\
\text { coefficient } \alpha_{\mathrm{SL}}\left(\mathrm{cm}^{-1}\right)\end{array}$} & \multirow{2}{*}{$\begin{array}{c}\text { InAs } \\
\text { thickness (nm) }\end{array}$} & \multirow{2}{*}{$\begin{array}{c}\text { InAsSb } \\
\text { thickness (nm) }\end{array}$} & \multirow{2}{*}{$\begin{array}{l}\mathrm{Sb} \text { mole } \\
\text { fraction }\end{array}$} & \multirow{2}{*}{$\begin{array}{c}\text { Optimal } \\
\text { or practical }\end{array}$} \\
\hline at $0 \mathrm{~K}$ & at $295 \mathrm{~K}$ & & & & & & \\
\hline 4.0 & 4.8 & 94.6 & 4721 & 1.884 & 1.396 & 0.211 & Optimal \\
\hline 5.0 & 6.3 & 66.3 & 3308 & 3.749 & 1.121 & 0.395 & Optimal \\
\hline 5.0 & 6.3 & 66.3 & 3308 & 3.748 & 1.162 & 0.384 & Practical \\
\hline 8.0 & 12.3 & 37.5 & 1871 & 5.780 & 1.160 & 0.550 & Optimal \\
\hline 8.0 & 12.1 & 34.8 & 1737 & 6.694 & 2.076 & 0.384 & Practical \\
\hline 10.0 & 18.0 & 31.3 & 1562 & 6.484 & 1.196 & 0.592 & Optimal \\
\hline 10.0 & 17.5 & 26.6 & 1327 & 8.465 & 2.625 & 0.384 & Practical \\
\hline 12.0 & 26.2 & 27.7 & 1382 & 6.970 & 1.220 & 0.620 & Optimal \\
\hline 12.0 & 25.1 & 21.2 & 1058 & 10.168 & 3.152 & 0.384 & Practical \\
\hline 15.0 & 47.3 & 24.5 & 1223 & 7.472 & 1.248 & 0.646 & Optimal \\
\hline 15.0 & 44.8 & 15.5 & 774 & 12.595 & 3.905 & 0.384 & Practical \\
\hline
\end{tabular}


Strain-balanced InAs/GaInSb.

\begin{tabular}{|c|c|c|c|c|c|c|c|}
\hline \multicolumn{2}{|c|}{ Detector wavelength $(\mu \mathrm{m})$} & \multirow{2}{*}{$\begin{array}{l}\text { Wavefunction } \\
\text { overlap square } \\
\left|\Psi_{h}^{*} \Psi_{e}\right|^{2}(\%)\end{array}$} & \multirow{2}{*}{$\begin{array}{c}\text { Absorption } \\
\text { coefficient } \alpha_{\mathrm{SL}}\left(\mathrm{cm}^{-1}\right)\end{array}$} & \multirow{2}{*}{$\begin{array}{l}\text { InAs thickness } \\
(\mathrm{nm})\end{array}$} & \multirow{2}{*}{$\begin{array}{l}\text { GaInSb thickness } \\
(\mathrm{nm})\end{array}$} & \multirow{2}{*}{$\begin{array}{l}\text { In mole } \\
\text { fraction }\end{array}$} & \multirow{2}{*}{$\begin{array}{c}\text { Optimal } \\
\text { or practical }\end{array}$} \\
\hline at $0 \mathrm{~K}$ & at $295 \mathrm{~K}$ & & & & & & \\
\hline 3.0 & 3.6 & 95.7 & 5501 & 1.488 & 0.502 & 0.297 & Practical \\
\hline 4.0 & 5.0 & 74.4 & 4226 & 3.239 & 0.491 & 0.676 & Optimal \\
\hline 4.0 & 5.1 & 71.3 & 4050 & 2.642 & 0.818 & 0.324 & Practical \\
\hline 5.0 & 6.7 & 55.5 & 3152 & 3.584 & 0.806 & 0.450 & Optimal \\
\hline 5.0 & 6.8 & 54.7 & 3107 & 3.275 & 1.015 & 0.324 & Practical \\
\hline 8.0 & 13.6 & 34.8 & 1977 & 4.623 & 1.177 & 0.396 & Optimal \\
\hline 8.0 & 13.7 & 34.5 & 1960 & 4.398 & 1.362 & 0.324 & Practical \\
\hline 10.0 & 20.6 & 29.3 & 1664 & 5.143 & 1.257 & 0.413 & Optimal \\
\hline 10.0 & 20.8 & 28.9 & 1642 & 4.848 & 1.502 & 0.324 & Practical \\
\hline 12.0 & 31.4 & 25.9 & 1471 & 5.551 & 1.299 & 0.432 & Optimal \\
\hline 12.0 & 31.8 & 25.5 & 1448 & 5.176 & 1.604 & 0.324 & Practical \\
\hline 15.0 & 65.6 & 22.9 & 1301 & 6.012 & 1.328 & 0.458 & Optimal \\
\hline 15.0 & 67.8 & 22.2 & 1261 & 5.535 & 1.715 & 0.324 & Practical \\
\hline
\end{tabular}

Strain-balanced InAs/InAsBi

\begin{tabular}{|c|c|c|c|c|c|c|c|}
\hline \multicolumn{2}{|c|}{ Detector wavelength $(\mu \mathrm{m})$} & \multirow{2}{*}{$\begin{array}{c}\text { Wavefunction } \\
\text { overlap square } \\
\left|\Psi_{h}^{*} \Psi_{e}\right|^{2}(\%)\end{array}$} & \multirow{2}{*}{$\begin{array}{c}\text { Absorption } \\
\text { coefficient } \alpha_{\mathrm{SL}}\left(\mathrm{cm}^{-1}\right)\end{array}$} & \multirow{2}{*}{$\begin{array}{l}\text { InAs thickness } \\
\text { (nm) }\end{array}$} & \multirow{2}{*}{$\begin{array}{c}\text { InAsBi } \\
\text { thickness (nm) }\end{array}$} & \multirow{2}{*}{$\begin{array}{l}\text { Bi mole } \\
\text { fraction }\end{array}$} & \multirow{2}{*}{$\begin{array}{l}\text { Optimal or } \\
\text { practical }\end{array}$} \\
\hline at $0 \mathrm{~K}$ & at $295 \mathrm{~K}$ & & & & & & \\
\hline 10.0 & 19.0 & 99.7 & 1410 & 0.500 & 0.760 & 0.113 & Practical \\
\hline 12.0 & 27.8 & 98.3 & 1093 & 0.427 & 4.083 & 0.075 & Optimal \\
\hline 12.0 & 27.9 & 98.2 & 1092 & 0.500 & 3.350 & 0.078 & Practical \\
\hline 15.0 & 51.5 & 91.6 & 1019 & 1.322 & 2.008 & 0.113 & Optimal \\
\hline
\end{tabular}

${ }^{1}$ H. Kroemer, Proc. IEEE 51, 1782 (1963).

${ }^{2}$ I. Vurgaftman, J. R. Meyer, and L. Ram-Mohan, J. Appl. Phys. 89, 5815 (2001).

${ }^{3}$ H. Okamoto and K. Oe, Jpn. J. Appl. Phys., Part 1 38, 1022 (1999).

${ }^{4}$ Z. M. Fang, K. Y. Ma, R. M. Cohen, and G. B. Stringfellow, J. Appl. Phys. 68, 1187 (1990).

${ }^{5}$ P. T. Webster, N. A. Riordan, C. Gogineni, S. Liu, J. Lu, X.-H. Zhao, D. J. Smith, Y.-H. Zhang, and S. R. Johnson, J. Vac. Sci. Technol. B 32, 02C120 (2014).

${ }^{6}$ L. Esaki and R. Tsu, IBM J. Res. Dev. 14, 61 (1970).

${ }^{7}$ N. J. Ekins-Daukes, K. Kawaguchi, and J. Zhang, Cryst. Growth Des. 2, 287 (2002)

${ }^{8}$ P. T. Webster, N. A. Riordan, S. Liu, E. H. Steenbergen, R. A. Synowicki, Y.-H. Zhang, and S. R. Johnson, Appl. Phys. Lett. 106, 061907 (2015).

${ }^{9}$ A. J. Shalindar, P. T. Webster, and S. R. Johnson, "Measurement of InAsBi mole fraction and InBi lattice constant using Rutherford backscattering spectrometry and X-ray diffraction,” J. Appl. Phys. (unpublished).

${ }^{10} 3 \mathrm{G}$ Series $10 \mathrm{C} 05$ system, Ircon Inc., Santa Cruz, CA, USA.

${ }^{11}$ VASE and IR-VASE systems, J. A. Woollam Co. Inc., Lincoln, NE, USA. ${ }^{12}$ WVASE software, J. A. Woollam Co. Inc., Lincoln, NE, USA.

${ }^{13}$ M. Schubert, Infrared Ellipsometry on Semiconductor Layer Structures: Phonons, Plasmons, and Polaritons (Springer, Heidelberg, 2004), p. 21.

${ }^{14}$ K. Alberi, J. Wu, W. Walukiewicz, K. M. Yu, O. D. Dubon, S. P. Watkins, C. X. Wang, X. Liu, Y.-J. Cho, and J. Furdyna, Phys. Rev. B 75, 045203 (2007).
${ }^{15}$ M. Gladysiewicz, R. Kudrawiec, and M. S. Wartak, J. Appl. Phys. 118, 055702 (2015).

${ }^{16}$ J. Wu, W. Shan, and W. Walukiewicz, Semicond. Sci. Technol. 17, 860 (2002).

${ }^{17}$ A. Janotti, S.-H. Wei, and S. B. Zhang, Phys. Rev. B 65, 115203 (2002).

${ }^{18}$ G. L. Bir and G. E. Pikus, Symmetry and Strain-Induced Effects in Semiconductors (Wiley, New York, 1974).

${ }^{19}$ S. L. Chuang, Physics of Optoelectronic Devices (Wiley, New York, 1995).

${ }^{20}$ P. T. Webster, N. A. Riordan, S. Liu, E. H. Steenbergen, R. A. Synowicki, Y.-H. Zhang, and S. R. Johnson, J. Appl. Phys. 118, 245706 (2015).

${ }^{21}$ A. P. Roth, W. J. Keeler, and E. Fortin, Can. J. Phys. 58, 560 (1980).

${ }^{22}$ R. de L. Kronig and W. G. Penney, Proc. R. Soc. Lond. A 130, 499 (1931).

${ }^{23}$ H.-S. Cho and P. R. Prucnal, Phys Rev. B 36, 3237 (1987).

${ }^{24}$ S.-H. Pan and S.-M. Feng, Phys. Rev. B 44, 5668 (1991).

${ }^{25}$ M. Kawabe, M. Kondo, N. Matsuura, and K. Yamamoto, Jpn. J. Appl. Phys., Part 2 22, L64 (1983).

${ }^{26}$ A. C. Gossard, Semiconductors and Semimetals (Academic Press, New York, 1994), Vol. 40, pp. 153-167.

${ }^{27}$ J. W. Matthews and A. E. Blakeslee, J. Cryst. Growth 27, 118 (1974).

${ }^{28}$ E. H. Steenbergen, B. C. Connelly, G. D. Metcalfe, H. Shen, M. Wraback, D. Lubyshev, Y. Qiu, J. M. Fastenau, A. W. K. Liu, S. Elhamri, O. O. Cellek, and Y.-H. Zhang, Appl. Phys. Lett. 99, 251110 (2011).

${ }^{29}$ The extrapolated $100 \%$ wavefunction overlap absorption coefficient is modified to account for unintentional Sb results reported in Ref. 20. This is discussed in detail in Section X of this work. 